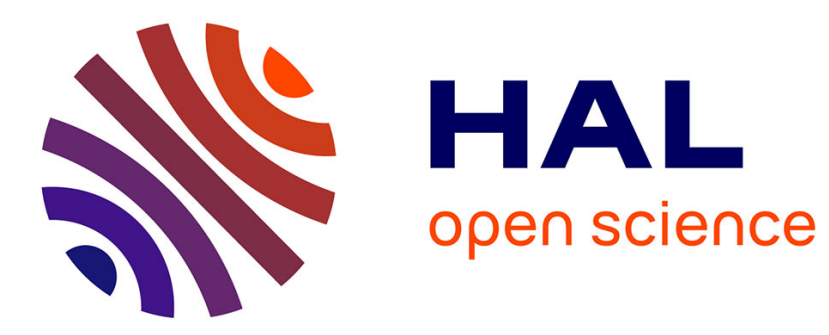

\title{
Obliquely Reflected Backward Stochastic Differential Equations
}

Jean-François Chassagneux, Adrien Richou

\section{To cite this version:}

Jean-François Chassagneux, Adrien Richou. Obliquely Reflected Backward Stochastic Differential Equations. Annales de l'Institut Henri Poincaré (B) Probabilités et Statistiques, In press. hal01761991

\section{HAL Id: hal-01761991 \\ https://hal.science/hal-01761991}

Submitted on 9 Apr 2018

HAL is a multi-disciplinary open access archive for the deposit and dissemination of scientific research documents, whether they are published or not. The documents may come from teaching and research institutions in France or abroad, or from public or private research centers.
L'archive ouverte pluridisciplinaire HAL, est destinée au dépôt et à la diffusion de documents scientifiques de niveau recherche, publiés ou non, émanant des établissements d'enseignement et de recherche français ou étrangers, des laboratoires publics ou privés. 


\title{
Obliquely Reflected Backward Stochastic Differential Equations
}

\author{
Jean-François Chassagneux \\ Laboratoire de Probabilités, Statistique et Modélisation \\ CNRS, UMR 8001, Université Paris Diderot \\ jean-francois.chassagneux@univ-paris-diderot.fr \\ Adrien Richou \\ Univ. Bordeaux, IMB, UMR 5251, F-33400 Talence, France. \\ adrien.richou@math.univ-bordeaux.fr
}

April 9, 2018

\begin{abstract}
In this paper, we study existence and uniqueness to multidimensional Reflected Backward Stochastic Differential Equations in an open convex domain, allowing for oblique directions of reflection. In a Markovian framework, combining a priori estimates for penalised equations and compactness arguments, we obtain existence results under quite weak assumptions on the driver of the BSDEs and the direction of reflection, which is allowed to depend on both $Y$ and $Z$. In a non Markovian framework, we obtain existence and uniqueness result for direction of reflection depending on time and $Y$. We make use in this case of stability estimates that require some smoothness conditions on the domain and the direction of reflection.
\end{abstract}

Key words: BSDE with oblique reflections

MSC Classification (2000): 93E20, 65C99, 60H30.

\section{Introduction}

In this paper, we study a class of BSDE whose solution is constrained to stay in an open convex domain, hereafter denoted $\mathcal{D}$. The "reflection" at the boundary of the domain is made along an oblique direction. Such equations are known as Obliquely Reflected BSDEs and they allow to represent the solution to some stochastic control problems. Precisely, let $(\Omega, \mathcal{F}, \mathbb{P})$ be a complete probability space and $\left(W_{t}\right)_{t \in[0, T]}$ a $k$-dimensional Brownian motion, defined on this space, whose natural filtration is denoted $\left(\mathcal{F}_{t}\right)_{t \in[0, T]}$. $\mathcal{P}$ is the $\sigma$-algebra generated by the progressively measurable processes on $[0, T] \times \Omega$. In the sequel, $T>0$ is a terminal time for the equation under consideration. In this 
paper, we are interested in the study of existence and uniqueness of a $\mathcal{P}$ - measurable solution $(Y, Z, \Phi)$ to the following equation

$$
\left\{\begin{array}{l}
(i) Y_{t}=\xi+\int_{t}^{T} f\left(s, Y_{s}, Z_{s}\right) \mathrm{d} s-\int_{t}^{T} H\left(s, Y_{s}, Z_{s}\right) \Phi_{s} \mathrm{~d} s-\int_{t}^{T} Z_{s} \mathrm{~d} W_{s}, 0 \leqslant t \leqslant T, \\
(\text { ii }) Y . \in \overline{\mathcal{D}} \text { a.s., } \quad \Phi . \in \partial \varphi(Y .) \mathrm{d} t \otimes \mathrm{d} \mathbb{P}-\text { a.e. }, \quad \int_{0}^{T}\left|\Phi_{s}\right| \mathbf{1}_{\left\{Y_{s} \notin \partial \mathcal{D}\right\}} \mathrm{d} s=0,
\end{array}\right.
$$

where $\partial \mathcal{D}$ is the boundary of the open convex domain $\mathcal{D}, \varphi$ the (convex) indicator function of $\mathcal{D}, \partial \varphi$ the subdifferential of $\varphi$ and $(f, H): \Omega \times[0, T] \times \mathbb{R}^{d} \times \mathbb{R}^{d \times k} \rightarrow$ $\left(\mathbb{R}^{d}, \mathbb{R}^{d \times d}\right)$ is a $\mathcal{P} \otimes \mathcal{B}\left(\mathbb{R}^{d} \times \mathbb{R}^{d \times k}\right)$-measurable random function. The terminal value $\xi$ is given as a parameter and belongs to $\mathscr{L}^{2}\left(\mathcal{F}_{T}\right)$, where for $p>0$ and a $\sigma$-algebra $\mathcal{B}, \mathscr{L}^{p}(\mathcal{B})$ is the space of $\mathcal{B}$-measurable random $R$ variable satisfying $\mathbb{E}\left[|R|^{p}\right]<+\infty$. Of course, we shall require some extra conditions to get an existence and uniqueness result. Classically, we will look for solution with the following integrability property: $(Y, Z, \Phi) \in \mathscr{S}^{2} \times \mathscr{H}^{2} \times \mathscr{H}^{2}$, where, for $p \in[1, \infty], \mathscr{H}^{p}$ is the set of progressively measurable process $V$ such that $\mathbb{E}\left[\left(\int_{0}^{T}\left|V_{t}\right|^{2} \mathrm{~d} t\right)^{\frac{p}{2}}\right]<+\infty$, and $\mathscr{S}^{p}$ is the set of continuous and adapted processes $U$ satisfying $\mathbb{E}\left[\sup _{t \in[0, T]}\left|U_{t}\right|^{p}\right]$. The main constraints on the couple $(Y, \Phi)$ are given in (1.1) $(i i)$. As already mentioned, the first one is that $Y$ takes its value in $\overline{\mathcal{D}}$, where $\mathcal{D}$ is a non-empty open convex subset of $\mathbb{R}^{d}$. The fact that $\Phi_{t} \in \partial \varphi\left(Y_{t}\right)$ imposes that $\Phi$ is directed along the outward normal of the convex domain, the important point being that in $(i)$ this direction is perturbed by the operator $H$ and we are thus dealing with an oblique direction of reflection. When 1.1) (i) is viewed backward in time, the process $\Phi$ or, more precisely $\Psi:=H(\cdot) \Phi$, is the process allowing $Y$ to stay in $\overline{\mathcal{D}}$. The condition $\int_{0}^{T}\left|\Phi_{t}\right| \mathbf{1}_{\left\{Y_{t} \notin \partial \mathcal{D}\right\}} \mathrm{d} t=0$ is then interpreted as a minimality condition, in the sense that $\Psi$ will be active only when $Y$ touches the boundary of the domain. This is of course one of the main ingredient to get uniqueness result for this kind of equation.

Let us now mention some known results about these equations. In the one dimensional case, they have been first studied in [6] for the - so called- simply reflected case and in [3] for the doubly reflected case. The literature on this specific form of equation has then grown very importantly due to their range of application, in particular in mathematical economics or stochastic control. The multidimensional case is only well understood in the case of normal reflection i.e. when the matrix-valued random function $H$ is equal to the identity, see [8]. The case of oblique direction of reflection has been only partially treated. Up until recently, only very specific cases have been considered for the couple $(H, \mathcal{D})$. In [22], the author studies the case of the reflection in an orthant with some restriction on the direction of oblique reflection and the driver $f$. Another case that has received a lot of attention is the setting of RBSDEs associated to "switching problems", see e.g. [13, 11, 1] and the references therein: the multidimensional domain has a specific form and the direction is along the axis, see also Section 4.1 for more details. In this case, structural conditions on $f$ are required to retrieve existence and uniqueness results also. This restriction are based on the technique of proof used to 
obtain the results and which is mainly based on a monotonic limit theorem à la Peng [21], in a multidimensional setting. To the best of our knowledge, the first attempt to treat the question of BSDEs with oblique reflection in full generality can be found in [7]. Unfortunately, their setting is still quite restrictive concerning $H$ and $f$.

To the best of our knowledge, there is no, up to now, satisfying global approach for the question of well-posedness of Obliquely Reflected BSDEs, especially when compared the case of forward SDEs, where existence and uniqueness results are obtained for oblique reflection and general domain, see e.g. the seminal paper [15].

Our goal in this paper is thus to prove existence and uniqueness for the RBSDEs (1.1) for generic $H$ and convex domain $\mathcal{D}$ without imposing any structural dependence condition on the driver $f$ of the equation. In this direction, we are able to obtain very general existence result in a Markovian setting, assuming only a weak domination property of the forward process, see Section 4. We also discuss there the non-uniqueness issue. In the general case of $\mathcal{P}$-measurable random coefficients $f, H$ and terminal condition $\xi$, we need to impose some smoothness assumptions on the domain, on $H$, which depend then only on the time and $y$ variables and on the terminal condition $\xi$. In this case, we obtain both existence and uniqueness for the solution of (1.1). Let us remark that our new results on Obliquely Reflected BSDEs allow us to treat some new optimal switching problems called "randomised switching problems" and introduced in the Section 5 of [2].

The main tool to obtain the existence result is to consider a sequence of penalised equation: for $n \in \mathbb{N}, t \in[0, T]$,

$$
Y_{t}^{n}=\xi+\int_{t}^{T} f\left(s, Y_{s}^{n}, Z_{s}^{n}\right) \mathrm{d} s-\int_{t}^{T} Z_{s}^{n} \mathrm{~d} W_{s}-\int_{t}^{T} H\left(s, Y_{s}^{n}, Z_{s}^{n}\right) \nabla \varphi_{n}^{M}\left(Y_{s}^{n}\right) d s
$$

where, for $y \in \mathbb{R}^{d}$, and some $M>0$,

$$
\varphi_{n}^{M}(y):=n \inf _{x \in \mathcal{D}} \theta_{M}(y-x) \quad \text { with } \quad \theta_{M}(h)=\left\{\begin{array}{rlll}
M|h|-\frac{M^{2}}{2} & \text { if } & |h|>M, \\
\frac{1}{2}|h|^{2} & \text { if } & |h| \leqslant M .
\end{array}\right.
$$

The key point is to obtain the convergence in a strong sense of $\left(Y^{n}\right)$ to some process $Y$ along with some a priori estimates on $\left(Z^{n}, \Phi^{n}\right)$. This will then allow to obtain the existence of some limiting process $(Z, \Phi)$ as well. In the setting of oblique direction of reflection, the question of uniqueness has generally to be investigated separately.

The first possible argument to obtain the convergence of $\left(Y^{n}\right)$ is to prove some monotonicity on the sequence to apply Peng's monotonic limit theorem [21]. In a multidimensional setting, this monotonicity is obtained under very restrictive structural condition on the coefficient. Nevertheless, it has been successfully used for the study of RBSDE associated to switching problem. Another possible argument is to invoke some fine compactness arguments and this is the approach followed in [7]. But, again some strong structural conditions are required to obtain convergence results in a weak setting. In this paper, we follow a similar approach in the Markovian setting, see Section 4 . At the heart of our proof, we use the paper [10], which was concerned with multidimensional (non-reflected) BSDEs with continuous only driver $f$. With this approach, in 
the Markovian setting, we are able to obtain existence result for $H$ that can depend on $Z$ and even be discontinuous. To the best of our knowledge, this is the first time such general setting is considered successfully. It has been brought to our attention that independantly from us, 4] has followed a similar approach to treat BSDEs associated to the classical switching problem in a more restrictive setting.

The last approach to obtain convergence of the sequence $\left(Y_{n}\right)$ is to show classically that it is a Cauchy sequence. This approach has been used in the case of muldimensional RBSDE when there is no perturbation $H$ of the direction of reflection, namely $H$ is the identity matrix of $\mathbb{R}^{d}$, in the seminal paper [8]. To obtain this result and a key stability estimate, authors of [8] use dramatically the convexity property of the domain linked with the normal reflection by applying Itô's formula to the Euclidean norm of the difference of two solutions. In our setting of general perturbation $H$, we cannot follow directly their proof. In order to retrieve the stability estimates, we modify the Euclidean norm to take into account the oblique reflection inspired by [15]. Unfortunately, this produces new terms that have to be controlled. The most difficult one is certainly the term linked to the quadratic variation of the martingale term in 1.1) (i) or 1.2). Let us emphasize that this term cannot be dealt with as one would do in the forward SDE case. Nevertheless, we are able to treat this term using BMO martingales estimates. This tool was already used with success to deal with quadratic BSDEs but, to the best of our knowledge, this approach is completely new in the setting of Reflected BSDEs. We are then able to obtain in the non-Markovian setting existence results when $(\mathcal{D}, H)$ satisfies some $C^{2}$ smoothness condition, with $H$ depending only on the time and $y$ variables. Let us note also that in this case the uniqueness result is obtained as an easy consequence of the stability estimate.

The rest of the paper is organised as follows. In the next Section, we present precisely our framework and the assumption made on the coefficients along with some discussions on these assumptions. We also prove the key a priori and stability estimates, that will be used later on. In Section 3, we present our first novel result on existence and uniqueness of Obliquely Reflected BSDEs in a regular setting for $(\mathcal{D}, H)$. In Section 4 , restricting to a Markovian framework, we extend our previous existence result assuming no regularity on $(\mathcal{D}, H)$ and allowing a dependence in $Z$ for the $H$ operator.

Notations We denote by $\varphi$ the indicator function of $\mathcal{D}$

$$
\varphi(y)= \begin{cases}0 & \text { if } y \in \overline{\mathcal{D}} \\ +\infty & \text { otherwise }\end{cases}
$$

and $\partial \varphi$ its subdifferential operator:

$$
\partial \varphi(y)= \begin{cases}\left\{\hat{y} \in \mathbb{R}^{d}: \hat{y} \cdot(z-y) \leqslant 0, \forall z \in \overline{\mathcal{D}}\right\} & \text { if } y \in \overline{\mathcal{D}} \\ \varnothing & \text { if } y \notin \overline{\mathcal{D}}\end{cases}
$$

In particular, $\partial \varphi(y)$ is the closed cone of outward normal to $\mathcal{D}$ at $y$ when $y \in \partial \mathcal{D}$ and $\partial \varphi(y)=\{0\}$ when $y \in \mathcal{D}$. Finally, we denote by $\mathfrak{P}$ the projection onto $\overline{\mathcal{D}}$ and by $\mathfrak{n}(y)$ the set of unit outward normal at $y \in \partial \mathcal{D}$. 
For a matrix $M$, we denote $M^{\dagger}$ its transpose.

We denote $\mathscr{B}^{2}$, the set of processes $V \in \mathscr{H}^{2}$, such that

$$
\|V\|_{\mathscr{B}^{2}}:=\left\|\sup _{t \in[0, T]} \mathbb{E}\left[\int_{t}^{T}\left|V_{s}\right|^{2} \mathrm{~d} s \mid \mathcal{F}_{t}\right]\right\|_{\mathscr{L}^{\infty}}^{\frac{1}{2}}<+\infty .
$$

Let us remark that $V \in \mathscr{B}^{2}$ means that the martingale $\int_{0}^{\delta_{s}} V_{s} \mathrm{~d} W_{s}$ is a BMO martingale and $\|V\|_{\mathscr{B}^{2}}$ is the BMO norm of $\int_{0}^{V_{s}} V_{s} \mathrm{~d} W_{s}$. We refer to [14 for further details about BMO martingales.

The set of continuous function from $[0, T]$ to $\mathbb{R}^{n}$ is denoted $\mathcal{C}\left([0, T], \mathbb{R}^{n}\right)$. For $x \in$ $\mathcal{C}\left([0, T], \mathbb{R}^{n}\right)$, we denote by $\|x\|_{\infty}:=\sup _{t \in[0, T]}\left|x_{t}\right|$, the sup-norm on this space.

\section{Setting and preliminary estimates}

In this section, we first introduce and discuss the main assumptions that will be used to obtain our existence and uniqueness results. In a second part, we give important a priori estimates and prove a key stability result, which is one of the novelty in our approach to solve Obliquely Reflected BSDEs.

\subsection{Framework}

The first minimal set of assumption that we consider here is the following.

\section{Assumption (A)}

i) $\xi$ is an $\mathcal{F}_{T}$-measurable random variable, $\mathbb{R}^{d}$-valued such that $\mathbb{E}\left[|\xi|^{2}\right]<+\infty$.

ii) $f: \Omega \times[0, T] \times \mathbb{R}^{d} \times \mathbb{R}^{d \times k} \rightarrow \mathbb{R}^{d}$ is a $\mathcal{P} \otimes \mathcal{B}\left(\mathbb{R}^{d} \times \mathbb{R}^{d \times k}\right)$-measurable function and there exists a non negative progressively measurable process $\alpha \in \mathscr{H}^{2}(\mathbb{R})$ and a constant $L$ such that

$$
|f(t, y, z)| \leqslant \alpha_{t}+L(|y|+|z|), \quad \forall(t, y, z) \in[0, T] \times \mathbb{R}^{d} \times \mathbb{R}^{d \times k} .
$$

iii) $H: \Omega \times[0, T] \times \mathbb{R}^{d} \times \mathbb{R}^{d \times k} \rightarrow \mathbb{R}^{d \times d}$ is a $\mathcal{P} \otimes \mathcal{B}\left(\mathbb{R}^{d} \times \mathbb{R}^{d \times k}\right)$-measurable function and there exists a constant $\eta>0$ such that, for any $(t, y, z) \in[0, T] \times \mathbb{R}^{d} \times \mathbb{R}^{d \times k}$

$$
\begin{aligned}
& H(t, \mathfrak{P}(y), z) v \cdot v \geqslant \eta, \quad v \in \mathfrak{n}(\mathfrak{P}(y)), \\
& |H(t, \mathfrak{P}(y), z)| \leqslant L .
\end{aligned}
$$

The above assumptions are too weak to obtain existence and uniqueness result in a general random framework. They will be used in Section 4 in a Markovian framework with their Markovian counterpart (AM). Nevertheless, it is possible to derive useful $a$ priori estimates in the general setting of (A). 
Remark 2.1. In applications, $H(t, \cdot, z)$ is usually specified only on the boundary $\partial \mathcal{D}$. The extension to $\mathbb{R}^{d} \backslash \overline{\mathcal{D}}$ is done easily by setting $H(t, y, z):=H(t, \mathfrak{P}(y), z)$. Moreover, if $H(t, \cdot, z)$ is a continuous and bounded function on $\partial \mathcal{D}$ it is possible to extend it to a continuous and bounded function on $\overline{\mathcal{D}}$. Indeed, $\overline{\mathcal{D}}$ is homeomorph to a set $S$ which is a half plane of $\mathbb{R}^{d}$ or $\mathbb{R}^{r} \times B^{d-r}$, with $0 \leqslant r \leqslant d$. Moreover, the boundary of $\mathcal{D}$ is sent to the boundary of $S$. Then we remark that the extension of $H(t, ., z)$ is straightforward when $\mathcal{D}=S$.

In the non-Markovian setting, our results require more smoothness and control on the parameters of the BSDE. We will then work under the following assumption.

\section{Assumption (SB)}

i) $\xi$ is an $\mathcal{F}_{T}$-measurable $\overline{\mathcal{D}}$-valued random variable and the martingale $\mathcal{Y}_{t}^{\xi}:=\mathbb{E}_{t}[\xi]=$ $\xi-\int_{t}^{T} \mathcal{Z}_{s}^{\xi} \mathrm{d} W_{s}, t \leqslant T$, is BMO (see [14] for further details on BMO martingales).

ii) $f: \Omega \times[0, T] \times \mathbb{R}^{d} \times \mathbb{R}^{d \times k} \rightarrow \mathbb{R}^{d}$ is a $\mathcal{P} \otimes \mathcal{B}\left(\mathbb{R}^{d} \times \mathbb{R}^{d \times k}\right)$-measurable function, there exists a constant $L>0$ such that, for all $\left(t, y, y^{\prime}, z, z^{\prime}\right) \in[0, T] \times \mathbb{R}^{d} \times \mathbb{R}^{d} \times \mathbb{R}^{d \times k} \times$ $\mathbb{R}^{d \times k}$

$$
\left|f(t, y, z)-f\left(t, y^{\prime}, z^{\prime}\right)\right| \leqslant L\left(\left|y-y^{\prime}\right|+\left|z-z^{\prime}\right|\right) .
$$

Moreover, the process $\theta^{\xi}:=f\left(\cdot, \mathcal{Y}^{\xi}, \mathcal{Z}^{\xi}\right)$ belongs to $\mathscr{B}^{2}$.

iii) The open convex domain $\mathcal{D}$ is given by a $\mathcal{C}^{2}\left(\mathbb{R}^{d}, \mathbb{R}\right)$ function $\phi$ with a bounded first derivative, namely $\mathcal{D}=\{\phi<0\}$ and $\partial \mathcal{D}=\{\phi=0\} . \phi$ is assumed . This function satisfies moreover

$$
|\phi(x)|=d(x, \partial \mathcal{D}) \quad \text { for } \quad x \in \mathcal{V} \cap\left(\mathbb{R}^{d} \backslash \overline{\mathcal{D}}\right) \text {, where } \mathcal{V} \text { is a neighbourhood of } \partial \mathcal{D} \text {. }
$$

iv) $H:[0, T] \times \mathbb{R}^{d} \rightarrow \mathbb{R}^{d \times d}$ is valued in the set of matrices $Q$ satisfying

$$
|Q| \leqslant L, \quad L|v|^{2} \geqslant v^{\dagger}\left(Q^{-1}\right)^{\dagger} v \geqslant \frac{1}{L}|v|^{2}, \forall v \in \mathbb{R}^{d} .
$$

$(t, y) \mapsto H(t, y)$ is a $\mathcal{C}^{0,1}$-function and $(t, y) \mapsto\left(H^{-1}\right)^{\dagger}(t, y)$ is a $\mathcal{C}^{1,2}$ function satisfying

$$
\left|\partial_{y} H\right|+\left|\left(H^{-1}\right)^{\dagger}\right|+\left|\partial_{t}\left(H^{-1}\right)^{\dagger}\right|+\left|\partial_{y}\left(H^{-1}\right)^{\dagger}\right|+\left|\partial_{y y}^{2}\left(H^{-1}\right)^{\dagger}\right| \leqslant \Lambda,
$$

for some positive $\Lambda$.

We first comment assumptions made on BSDE parameters.

Remark 2.2. i) Let us observe that under the BMO condition, there exists $\mu^{\xi}>0$, such that $\mathbb{E}\left[e^{\mu^{\xi} \sup _{t \in[0, T]}\left|\mathcal{Y}_{t}^{\xi}\right|}\right]<\infty$ and that $\left\|\mathcal{Z}^{\xi}\right\|_{\mathscr{B}^{2}}<\infty$, see e.g. [14]. For later use, we define

$$
\sigma^{\xi}:=\mathbb{E}\left[e^{\mu^{\xi} \sup _{t \in[0, T]}\left|\mathcal{Y}_{t}^{\xi}\right|}\right]+\left\|\mathcal{Z}^{\xi}\right\|_{\mathscr{B}^{2}}+\left\|\theta^{\xi}\right\|_{\mathscr{B}^{2}}<\infty .
$$


ii) Condition (SB)(ii) is a mix of the property of $\xi, f$ and the domain $\mathcal{D}$. In many applications, it will be straightforward to check. For example, it is trivially satisfied in the following cases:

(a) $\sup _{y \in \mathcal{D}} f(s, y, z) \leqslant C$, for some $C>0$;

(b) $\xi \in \mathscr{L}^{\infty}\left(\mathcal{F}_{T}\right)$;

(c) $\mathcal{D}$ is a bounded domain.

iii) If (SB) holds, then $(\boldsymbol{A})$ holds as well. Indeed, one can set $\alpha:=L\left(\left|\mathcal{Y}^{\xi}\right|+\left|\mathcal{Z}^{\xi}\right|+\left|\theta^{\xi}\right|\right)$.

We now discuss the various assumptions made on $H$ and the domain $\mathcal{D}$.

Remark 2.3. i) The function $\phi$ can be constructed as in e.g. [8] Section 2.4 if the convex domain $\mathcal{D}$ is $\mathcal{C}^{2}$. From $(S B)($ iii), it follows that $\partial \phi(x)$ (resp. $\mathfrak{n}(x)$ ) is the outward normal (resp. unit outward normal) of $\mathcal{D}$ at a point $x \in \partial \mathcal{D}$. Moreover, since $\mathcal{D}$ is convex, $\phi$ is convex on $\mathbb{R}^{d} \backslash \mathcal{D}$ and thus, $\partial_{x x}^{2} \phi$ is a positive semi-definite matrix on this domain. Let us also observe that the application $\mathfrak{P}: \mathbb{R}^{d} \backslash \overline{\mathcal{D}} \rightarrow \partial \mathcal{D}$ is $\mathcal{C}^{2}$

ii) The matrix $H$ defines on $\partial \mathcal{D}$ a unit vector field $\nu$ in the following way

$$
\tilde{\nu}(t, y):=H(t, y) \mathfrak{n}(y) \text { and } \nu(t, y):=\frac{\tilde{\nu}(t, y)}{|\tilde{\nu}(t, y)|}, \quad \text { for } y \in \partial \mathcal{D},
$$

which represents the oblique direction of reflection. Then, 2.2 rewrites as

$$
\langle\tilde{\nu}(t, y), \mathfrak{n}(y)\rangle \geqslant \eta, \quad \text { for } y \in \partial \mathcal{D} .
$$

In applications, it is generally the case that only the smooth vector field $\nu$ is given on $\partial \mathcal{D}$. Following [15], it is possible to construct $H$ satisfying $(S B)(i v)$ on $\partial \mathcal{D}$ and then to extend it on $\overline{\mathcal{D}}$ under $(S B)($ iii) using classical extension results, see e.g. [9].

We now introduce a class of terminal conditions that are admissible for the purpose of our work, in the sense that we can obtain an existence and uniqueness result for this class.

Definition 2.1. For $\beta>0$, the class $\mathfrak{T}_{\beta}$ is the subset of $\xi \in \mathscr{L}^{2}\left(\mathcal{F}_{T}\right)$ satisfying: there exists $\lambda_{\xi}>\beta$, such that

$$
\mathbb{E}\left[e^{\lambda_{\xi} \int_{0}^{T}\left|\mathcal{Z}_{s}^{\xi}\right|^{2} \mathrm{~d} s}\right]<\infty
$$

where $\mathcal{Z}^{\xi}$ is given by the martingale representation theorem applied to $\mathcal{Y}_{t}^{\xi}:=\mathbb{E}_{t}[\xi]=$ $\xi-\int_{t}^{T} \mathcal{Z}_{s}^{\xi} \mathrm{d} W_{s}, t \leqslant T$.

We study the class $\mathfrak{T}_{\beta}$ in Section 2.4. Especially, we exhibit some specific elements of this class that are quite useful for applications. 
Remark 2.4. In the following, we will use in proofs the notation " $C$ " to denote a generic constant that may change from line to line and that depends in an implicit way on $T, L$ and $\eta$. We shall denote it $C_{\theta}$, if it depends on an extra parameters $\theta$. In the statement of the results, we prefer the notation "c" and the dependence upon any extra parameters on top of $T, L$ and $\eta$, will also be made clear.

\section{$2.2 \quad$ A priori estimates}

In this section, we prove some a priori control on the solution $(Y, Z, \Phi) \in \mathscr{S}^{2} \times \mathscr{H}^{2} \times \mathscr{H}^{2}$ to the following generalised BSDE

$$
Y_{t}=\xi+\int_{t}^{T} f\left(s, Y_{s}, Z_{s}\right) d s-\int_{t}^{T} H\left(s, Y_{s}, Z_{s}\right) \Phi_{s} d s-\int_{t}^{T} Z_{s} d W_{s}, \quad 0 \leqslant t \leqslant T .
$$

Importantly, we assume that $(Y, Z, \Phi)$ satisfies the following structural condition:

$$
\mathbb{E}_{t}\left[\int_{t}^{T}\left|\Phi_{s}\right|^{2} \mathrm{~d} s\right] \leqslant K \mathbb{E}_{t}\left[\int_{t}^{T}\left|f\left(s, Y_{s}, Z_{s}\right)\right|^{2} \mathrm{~d} s\right], \text { for some } K>0
$$

Equation 2.11) encompasses both the obliquely reflected BSDE (1.1) and its penalised approximation given in equation (1.2). The key point for these two equations will then be to prove that their solutions satisfy condition 2.12).

Our first estimate is quite classical.

Lemma 2.1. Assume (A). Let $(Y, Z, \Phi) \in \mathscr{S}^{2} \times \mathscr{H}^{2} \times \mathscr{H}^{2}$ be a solution to (2.11) with condition 2.12 holding true. Then, for some $c:=c(K)$,

$$
\sup _{t \in[0, T]} \mathbb{E}\left[\left|Y_{t}\right|^{2}\right]+\mathbb{E}\left[\int_{0}^{T}\left|Z_{s}\right|^{2} \mathrm{~d} s\right] \leqslant c \mathbb{E}\left[|\xi|^{2}+\int_{0}^{T}\left|\alpha_{s}\right|^{2} d s\right] .
$$

Proof. We apply Itô's formula to $|Y|^{2}$ to obtain

$$
\left|Y_{t}\right|^{2}+\int_{t}^{T}\left|Z_{s}\right|^{2} \mathrm{~d} s=2 \int_{t}^{T} Y_{s} f\left(s, Y_{s}, Z_{s}\right) \mathrm{d} s-2 \int_{t}^{T} Y_{s} H\left(s, Y_{s}, Z_{s}\right) \Phi_{s} \mathrm{~d} s-2 \int_{t}^{T} Y_{s} Z_{s} \mathrm{~d} W_{s} .
$$

We observe, thanks to the square integrability of $Y$ and $Z$ that $\int_{0}^{\cdot} Y_{s} Z_{s} \mathrm{~d} W_{s}$ is a true martingale. This yields,

$$
\mathbb{E}\left[\left|Y_{t}\right|^{2}+\int_{t}^{T}\left|Z_{s}\right|^{2} \mathrm{~d} s\right]=\mathbb{E}\left[|\xi|^{2}+2 \int_{t}^{T} Y_{s} f\left(s, Y_{s}, Z_{s}\right) \mathrm{d} s-2 \int_{t}^{T} Y_{s} H\left(s, Y_{s}, Z_{s}\right) \Phi_{s} \mathrm{~d} s\right] .
$$

We thus compute, using 2.12 and Assumption (A)(ii), the boundedness of $H$ and Young's inequality, for some $\epsilon \in(0,1)$,

$$
\begin{aligned}
\mathbb{E}\left[\int_{t}^{T} Y_{s} H\left(s, Y_{s}, Z_{s}\right) \Phi_{s} \mathrm{~d} s\right] & \leqslant C_{K} \mathbb{E}\left[\int_{t}^{T}\left(\frac{1}{\epsilon}\left|Y_{s}\right|^{2}+\epsilon\left|\Phi_{s}\right|^{2}\right) \mathrm{d} s\right] \\
& \leqslant C_{K} \mathbb{E}\left[\int_{t}^{T}\left(\frac{1}{\epsilon}\left|Y_{s}\right|^{2}+\epsilon\left|Z_{s}\right|^{2}+\left|\alpha_{s}\right|^{2}\right) \mathrm{d} s\right] .
\end{aligned}
$$


Similarly, we get

$$
\mathbb{E}\left[\int_{t}^{T} Y_{s} f\left(s, Y_{s}, Z_{s}\right) \mathrm{d} s\right] \leqslant C \mathbb{E}\left[\int_{t}^{T}\left(\frac{1}{\epsilon}\left|Y_{s}\right|^{2}+\epsilon\left|Z_{s}\right|^{2}+\left|\alpha_{s}\right|^{2}\right) \mathrm{d} s\right] .
$$

For $\epsilon$ small enough and using Gronwall Lemma, we deduce

$$
\mathbb{E}\left[\left|Y_{t}\right|^{2}+\int_{t}^{T}\left|Z_{s}\right|^{2} \mathrm{~d} s\right] \leqslant C_{K} \mathbb{E}\left[|\xi|^{2}+\int_{t}^{T}\left|\alpha_{s}\right|^{2} \mathrm{~d} s\right] .
$$

The following proposition refines the previous estimates in the smooth setting of Assumption (SB). It will also allow to use the stability result proved in the next section. Interestingly, it shows that most of the properties of the martingale $\mathcal{Y}^{\xi}$ are transferred to the non-linear process given in equation (2.11).

Proposition 2.1. Assume that (SB) holds. Let $(Y, Z, \Phi) \in \mathscr{S}^{2} \times \mathscr{H}^{2} \times \mathscr{H}^{2}$ be a solution to 2.11) with condition 2.12 in force. Then, the following holds

i) $(Y, Z, \Phi) \in \mathscr{S}^{2} \times \mathscr{B}^{2} \times \mathscr{B}^{2}$ with, for some $c:=c\left(K, \sigma^{\xi}\right)$,

$$
\mathbb{E}\left[e^{\mu^{\xi} \sup _{t \in[0, T]}\left|Y_{t}\right|}\right]+\|\Phi\|_{\mathscr{B}^{2}}+\|Z\|_{\mathscr{B}^{2}} \leqslant c
$$

and, for all $b>0$ and some $c^{\prime}:=c^{\prime}\left(b, K, \sigma^{\xi}\right)$

$$
\mathbb{E}\left[e^{b \int_{0}^{T}\left(\left|\Phi_{s}\right|+\left|Z_{t}-\mathcal{Z}_{t}^{\xi}\right|+\left|\theta_{t}^{\xi}\right|\right) \mathrm{d} s}\right] \leqslant c^{\prime} .
$$

ii) Moreover, if $\xi \in \mathfrak{T}_{\beta}$, for some $\beta>0$, then there exists $\Theta \in \mathscr{H}^{2}$ such that, for all non negative increasing process $\gamma$ satisfying $\mathbb{E}\left[\left|\gamma_{T}\right|^{p}\right]<\infty$ for some $p>1$ (depending on $\gamma)$, we have for all $t \in[0, T]$

$$
\mathbb{E}_{t}\left[\int_{t}^{T} \gamma_{s}\left|Z_{s}\right|^{2} \mathrm{~d} s\right] \leqslant \mathbb{E}_{t}\left[\int_{t}^{T} \gamma_{s}\left|\Theta_{s}\right| \mathrm{d} s\right]<+\infty
$$

and for some $\lambda \in\left(\beta, \lambda^{\xi}\right)$ and $c:=c\left(K, \sigma^{\xi}, \lambda\right)$,

$$
\mathbb{E}\left[e^{\lambda \int_{0}^{T}\left|\Theta_{t}\right| \mathrm{d} t}\right] \leqslant c
$$

Proof. An important step to obtain our estimates below is to compare the BSDE $(Y, Z)$ with the martingale $\mathcal{Y}^{\xi}$. To this end, we introduce for this proof $\Delta Y:=Y-\mathcal{Y}^{\xi}$ and $\Delta Z=Z-\mathcal{Z}^{\xi}$.

1.a We apply Itô's formula to $|\Delta Y|^{2}$ to obtain

$$
\left|\Delta Y_{t}\right|^{2}+\int_{t}^{T}\left|\Delta Z_{s}\right|^{2} \mathrm{~d} s=2 \int_{t}^{T} \Delta Y_{s} f\left(s, Y_{s}, Z_{s}\right) \mathrm{d} s-2 \int_{t}^{T} \Delta Y_{s} H\left(s, Y_{s}\right) \Phi_{s} \mathrm{~d} s-2 \int_{t}^{T} \Delta Y_{s} \Delta Z_{s} \mathrm{~d} W_{s} .
$$


We observe, thanks to the square integrability of $\Delta Y$ and $\Delta Z$ that $\int_{0}^{\cdot} \Delta Y_{s} \Delta Z_{s} \mathrm{~d} W_{s}$ is a true martingale. This yields, for all $r \leqslant t$,

$$
\mathbb{E}_{r}\left[\left|\Delta Y_{t}\right|^{2}+\int_{t}^{T}\left|\Delta Z_{s}\right|^{2} \mathrm{~d} s\right]=2 \mathbb{E}_{r}\left[\int_{t}^{T} \Delta Y_{s} f\left(s, Y_{s}, Z_{s}\right) \mathrm{d} s-\int_{t}^{T} \Delta Y_{s} H\left(s, Y_{s}\right) \Phi_{s} \mathrm{~d} s\right]
$$

We thus compute, using (2.12), the Lipschitz continuity of $f$, the boundedness of $H$ and Young's inequality, for all $r \leqslant t$ and some $\epsilon \in(0,1)$

$$
\begin{aligned}
\mathbb{E}_{r}\left[\int_{t}^{T} \Delta Y_{s} H\left(s, Y_{s}\right) \Phi_{s} \mathrm{~d} s\right] & \leqslant C_{K} \mathbb{E}_{r}\left[\int_{t}^{T}\left(\frac{1}{\epsilon}\left|\Delta Y_{s}\right|^{2}+\epsilon\left|\Phi_{s}\right|^{2}\right) \mathrm{d} s\right] \\
& \leqslant C_{K} \mathbb{E}_{r}\left[\int_{t}^{T}\left(\frac{1}{\epsilon}\left|\Delta Y_{s}\right|^{2}+\epsilon\left|\Delta Z_{s}\right|^{2}+\left|\theta_{s}^{\xi}\right|^{2}\right) \mathrm{d} s\right] .
\end{aligned}
$$

Similarly, we obtain

$$
\mathbb{E}_{r}\left[\int_{t}^{T} \Delta Y_{s} f\left(s, Y_{s}, Z_{s}\right) \mathrm{d} s\right] \leqslant C \mathbb{E}_{r}\left[\int_{t}^{T}\left(\frac{1}{\epsilon}\left|\Delta Y_{s}\right|^{2}+\epsilon\left|\Delta Z_{s}\right|^{2}+\left|\theta_{s}^{\xi}\right|^{2}\right) \mathrm{d} s\right] .
$$

Combining the last two estimates with (2.18), setting $\epsilon$ small enough and using Gronwall Lemma, we get for all $r \leqslant t$

$$
\mathbb{E}_{r}\left[\left|\Delta Y_{t}\right|^{2}+\frac{1}{2} \int_{t}^{T}\left|\Delta Z_{s}\right|^{2} \mathrm{~d} s\right] \leqslant C_{K} \mathbb{E}_{r}\left[\int_{t}^{T}\left|\theta_{s}^{\xi}\right|^{2} \mathrm{~d} s\right] .
$$

1.b Setting $r=t$ in the previous inequality, we have

$$
\sup _{t \in[0, T]}\left|\Delta Y_{t}\right|^{2}+\|\Delta Z\|_{\mathscr{B}^{2}}^{2} \leqslant C_{K, \sigma \xi}
$$

from which we straightforwardly deduce

$$
\begin{aligned}
\mathbb{E}\left[e^{\mu^{\xi} \sup _{t \in[0, T]}\left|Y_{t}\right|}\right] & \leqslant \mathbb{E}\left[e^{\mu^{\xi} \sup _{t \in[0, T]}\left(\left|\Delta Y_{t}\right|+\left|\mathcal{Y}_{t}^{\xi}\right|\right)}\right] \leqslant C_{K, \sigma^{\xi}} \\
\text { and }\|Z\|_{\mathscr{B}^{2}} & \leqslant\left\|\mathcal{Z}^{\xi}\right\|_{\mathscr{B}^{2}}+\|\Delta Z\|_{\mathscr{B}^{2}} \leqslant C_{K, \sigma^{\xi}} .
\end{aligned}
$$

Combining (2.19) with 2.12 , we obtain

$$
\|\Phi\|_{\mathscr{B}^{2}} \leqslant C_{K, \sigma^{\xi}} .
$$

This concludes the proof of (2.14).

2.a We denote $\mathfrak{R}:=|\Phi|+|\Delta Z|+\left|\theta^{\xi}\right|$. For all $b>0$, we use Young inequality to get

$$
\mathbb{E}\left[e^{b \int_{0}^{T} \Re_{s} \mathrm{~d} s}\right] \leqslant e^{\frac{b^{2} T}{\varepsilon}} \mathbb{E}\left[e^{\varepsilon \int_{0}^{T}\left|\Re_{s}\right|^{2} \mathrm{~d} s}\right]
$$


for all $\varepsilon>0$. Then, by setting $\varepsilon=\left(1+4\|\Phi\|_{\mathscr{B}^{2}}^{2}+4\|\Delta Z\|_{\mathscr{B}^{2}}^{2}+4\left\|\theta^{\xi}\right\|_{\mathscr{B}^{2}}^{2}\right)^{-1}$ we compute, for all $r \in[0, T]$,

$$
\mathbb{E}_{r}\left[\int_{r}^{T} \varepsilon\left|\Re_{s}\right|^{2} \mathrm{~d} s\right] \leqslant 3 \varepsilon \mathbb{E}_{r}\left[\int_{r}^{T}\left|\Phi_{s}\right|^{2}+\left|\Delta Z_{s}\right|^{2}+\left|\theta_{s}^{\xi}\right|^{2} \mathrm{~d} s\right] \leqslant \frac{3}{4} .
$$

Going back to 2.23 and applying the John-Nirenberg formula, see Theorem 2.2 in [14], we obtain

$$
\mathbb{E}\left[e^{b \int_{0}^{T} \Re_{s} \mathrm{~d} s}\right] \leqslant C_{K, \sigma^{\xi}, b},
$$

which proves (2.15).

2.b Applying Itô's formula to $\gamma \cdot|\Delta Y|^{2}$, on $[t, T]$, we compute,

$$
\begin{aligned}
\gamma_{t}\left|\Delta Y_{t}\right|^{2}+\int_{t}^{T} \gamma_{s}\left|\Delta Z_{s}\right|^{2} \mathrm{~d} s+\int_{t}^{T}\left|\Delta Y_{s}\right|^{2} \mathrm{~d} \gamma_{s}= & 2 \int_{t}^{T} \gamma_{s} \Delta Y_{s} f\left(s, Y_{s}, Z_{s}\right) \mathrm{d} s \\
& -2 \int_{t}^{T} \gamma_{s} \Delta Y_{s} H\left(s, Y_{s}\right) \Phi_{s} \mathrm{~d} s-2 \int_{t}^{T} \gamma_{s} \Delta Y_{s} \Delta Z_{s} \mathrm{~d} W_{s} .
\end{aligned}
$$

Let us observe that the local martingale $\int_{0}^{\cdot} \gamma_{t} \Delta Y_{t} \Delta Z_{t} \mathrm{~d} W_{t}$ is a true martingale. Indeed, we compute, using Burkholder-Davis-Gundy inequality,

$$
\begin{aligned}
\mathbb{E}\left[\sup _{s \in[0, T]}\left|\int_{0}^{s} \gamma_{t} \Delta Y_{t} \Delta Z_{t} \mathrm{~d} W_{t}\right|\right] & \leqslant C \mathbb{E}\left[\left(\int_{0}^{T}\left|\gamma_{t} \Delta Y_{t} \Delta Z_{t}\right|^{2} \mathrm{~d} t\right)^{\frac{1}{2}}\right] \\
& \leqslant C_{K, \sigma \xi} \mathbb{E}\left[\left|\gamma_{T}\right|\left(\int_{0}^{T}\left|\Delta Z_{t}\right|^{2} \mathrm{~d} t\right)^{\frac{1}{2}}\right]
\end{aligned}
$$

where we used (2.19) for the last inequality. Using Hölder inequality, denoting $q$ the conjugate exponent of $p$, we get

$$
\mathbb{E}\left[\sup _{s \in[0, T]}\left|\int_{0}^{s} \gamma_{t} \Delta Y_{t} \Delta Z_{t} \mathrm{~d} W_{t}\right|\right] \leqslant C_{K, \sigma^{\xi}, p} \mathbb{E}\left[\left|\gamma_{T}\right|^{p}\right]^{\frac{1}{p}} \mathbb{E}\left[\left(\int_{0}^{T}\left|\Delta Z_{t}\right|^{2} \mathrm{~d} t\right)^{\frac{q}{2}}\right]^{\frac{1}{q}} .
$$

From the energy inequality, c.f. (VI.109.7) in [5], we have that

$$
\mathbb{E}\left[\left(\int_{0}^{T}\left|\Delta Z_{t}\right|^{2} \mathrm{~d} t\right)^{\frac{\lceil q\rceil}{2}}\right] \leqslant C_{q}\|\Delta Z\|_{\mathscr{B}^{2}}^{[q]} .
$$

We thus deduce

$$
\mathbb{E}\left[\sup _{s \in[0, T]}\left|\int_{0}^{s} \gamma_{t} \Delta Y_{t} \Delta Z_{t} \mathrm{~d} W_{t}\right|\right]<\infty
$$


Since $\gamma$ is non-decreasing, we then compute, using 2.25, 2.12 and the Lipschitz continuity of $f$,

$$
\mathbb{E}_{t}\left[\int_{t}^{T} \gamma_{s}\left|\Delta Z_{s}\right|^{2} \mathrm{~d} s\right] \leqslant \mathbb{E}_{t}\left[\int_{t}^{T} \gamma_{s} \Xi_{s} \mathrm{~d} s\right]<\infty,
$$

where we set $\Xi:=C_{K, \sigma \xi}(1+\mathfrak{R})$ recalling that $\Delta Y$ is bounded by 2.19$)$ and $\mathfrak{R}$ is defined in step 2.a. Using (2.24) we compute

$$
\mathbb{E}\left[e^{b \int_{0}^{T}\left|\Xi_{s}\right| \mathrm{d} s}\right] \leqslant C_{K, \sigma^{\xi}, b},
$$

for all $b>0$.

2.c We set $\lambda=(1+\epsilon) \beta$ with $\epsilon>0$ such that $(1+\epsilon)^{2} \beta \leqslant \lambda_{\xi}$, recalling Definition 2.1. Now we define

$$
\Theta:=(1+\epsilon)\left|\mathcal{Z}^{\xi}\right|^{2}+\left(1+\frac{1}{\epsilon}\right) \Xi .
$$

We observe that $\mathbb{E}_{t}\left[\int_{t}^{T} \gamma_{s}\left|\Theta_{s}\right|^{2} \mathrm{~d} s\right]<\infty$ : this follows from 2.26 and the fact that

$$
\mathbb{E}_{t}\left[\int_{t}^{T} \gamma_{s}\left|\mathcal{Z}_{s}^{\xi}\right|^{2} \mathrm{~d} s\right]<\infty
$$

This last inequality is simply obtained by applying Itô's formula to $\gamma\left|\mathcal{Y}^{\xi}\right|^{2}$ which yields

$$
\mathbb{E}\left[\int_{0}^{T} \gamma_{s}\left|\mathcal{Z}_{s}^{\xi}\right|^{2} \mathrm{~d} s\right] \leqslant \mathbb{E}\left[\gamma_{T}|\xi|^{2}\right] \leqslant\left\|\gamma_{T}\right\|_{\mathscr{L}^{p}}\left\||\xi|^{2}\right\|_{\mathscr{L}^{q}}<\infty .
$$

From the definition of $\Theta$, we have that, for $t \leqslant T$,

$$
\mathbb{E}_{t}\left[\int_{t}^{T} \gamma_{s} \Theta_{s} \mathrm{~d} s\right] \geqslant(1+\epsilon) \mathbb{E}_{t}\left[\int_{t}^{T} \gamma_{s}\left|\mathcal{Z}_{s}^{\xi}\right|^{2} \mathrm{~d} s\right]+\left(1+\frac{1}{\epsilon}\right) \mathbb{E}_{t}\left[\int_{t}^{T} \gamma_{s}\left|\Delta Z_{s}\right|^{2} \mathrm{~d} s\right]
$$

where we used (2.26). Then it follows from Young's inequality,

$$
\mathbb{E}_{t}\left[\int_{t}^{T} \gamma_{s}\left|Z_{s}\right|^{2} \mathrm{~d} s\right] \leqslant C \mathbb{E}_{t}\left[\int_{t}^{T} \gamma_{s} \Theta_{s} \mathrm{~d} s\right]<\infty,
$$

which proves 2.16). Finally, we compute using Hölder's inequality,

$$
\mathbb{E}\left[e^{(1+\epsilon) \beta \int_{0}^{T} \Theta_{s} \mathrm{~d} s}\right] \leqslant C \mathbb{E}\left[e^{(1+\epsilon)^{2} \beta \int_{0}^{T}\left|\mathcal{Z}_{s}^{\xi}\right|^{2} \mathrm{~d} s}\right]^{\frac{1}{1+\epsilon}} \mathbb{E}\left[e^{\left(1+\frac{1}{\epsilon}\right)^{2} \beta \int_{0}^{T}\left|\Xi_{s}\right| \mathrm{d} s}\right]^{\frac{\epsilon}{1+\epsilon}}
$$

and using the fact that $\xi \in \mathfrak{T}_{\beta}$ and (2.27), we obtain (2.17) with $\lambda=(1+\epsilon) \beta$.

Let us remark the following result, that will be useful in the next section.

Corollary 2.1. Assume that $(\boldsymbol{S B})$ holds. Let $(Y, Z, \Phi) \in \mathscr{S}^{2} \times \mathscr{H}^{2} \times \mathscr{H}^{2}$ be a solution to (2.11) with condition (2.12) in force and assume moreover that $\xi \in \mathscr{L}^{\infty}\left(\mathcal{F}_{T}\right)$ then $Y$ is bounded, namely for some $c:=c\left(K, \sigma^{\xi},\|\xi\|_{\mathscr{L}^{\infty}}\right)$, we have

$$
\sup _{t \in[0, T]}\left|Y_{t}\right| \leqslant c .
$$

Proof. We observe that $\left|\mathcal{Y}_{t}^{\xi}\right| \leqslant\|\xi\|_{\mathscr{L}^{\infty}}$ and then conclude using (2.19). 


\section{$2.3 \quad$ A stability result}

In this section, we prove a key estimate for the difference of two solutions of the generalised BSDE (2.11) satisfying (2.12). For $i \in\{1,2\}$, we denote $\left({ }^{i} Y,{ }^{i} Z,{ }^{i} \Phi\right)$ the solutions associated to parameters $\left({ }^{i} \xi,{ }^{i} f\right)$ and we furthermore assume that

$$
{ }^{i} \Phi . \in \partial \varphi\left(\mathfrak{P}\left({ }^{i} Y .\right)\right) \mathrm{d} \mathbb{P} \otimes \mathrm{d} t-\text { a.e. } \quad \text { and } \quad \int_{0}^{T}\left|{ }^{i} \Phi_{t}\right| \mathbf{1}_{\left\{{ }^{i} Y_{t} \in \mathcal{D}\right\}} \mathrm{d} t=0 .
$$

Remark 2.5. The above assumption allows us to cover both cases of equation (1.1) and equation 1.2.

We now define $\delta Y={ }^{1} Y-{ }^{2} Y, \delta Z={ }^{1} Z-{ }^{2} Z, \delta \Psi={ }^{1} \Psi-{ }^{2} \Psi$, where ${ }^{i} \Psi=H\left(\cdot \cdot{ }^{i} Y\right){ }^{i} \Phi$ and $\delta f={ }^{1} f\left(\cdot,{ }^{1} Y,{ }^{1} Z\right)-{ }^{2} f\left(\cdot,{ }^{2} Y,{ }^{2} Z\right)$. We have the following key result for our work.

Proposition 2.2. Assume that $(\boldsymbol{S B})$ holds. There exist two increasing functions $\mathfrak{B}(\cdot)$ and $\mathfrak{A}(\cdot)$ from $(0, \infty)$ to $(0, \infty)$, such that for all ${ }^{1} \xi$ belonging to $\mathfrak{T}_{\mathfrak{B}(\Lambda)}$, setting

$$
\Gamma_{t}:=e^{\mathfrak{A}(\Lambda) t+\int_{0}^{t} \mathfrak{B}(\Lambda)\left\{\Theta_{s}^{1}+\Theta_{s}^{\Phi}+\Theta_{s}^{f}\right\} \mathrm{d} s}
$$

where $\Theta^{\Phi}:=\left|{ }^{1} \Phi\right|+\left|{ }^{2} \Phi\right|, \Theta^{f}:=\left|{ }^{1} Z-\mathcal{Z}^{1} \xi\right|+\left|\theta^{1} \xi\right|$, and $\Theta^{1}$ is given by Proposition 2.1 (ii) applied to the BSDE with parameters $\left({ }^{1} \xi,{ }^{1} f\right)$, we have,

i) $\mathbb{E}\left[\left|\Gamma_{T}\right|^{p}\right]<c$ for some $p:=p(\Lambda)>1$ and $c:=c\left(K, \Lambda, \sigma^{1 \xi}, \sigma^{2 \xi}\right)$;

ii) for some $c^{\prime}:=c^{\prime}\left(K, \Lambda, \sigma^{1 \xi}, \sigma^{2} \xi\right)$, and for all $t \leqslant T$,

$$
\begin{aligned}
\left|\delta Y_{t}\right|^{2}+\mathbb{E}_{t}\left[\int_{t}^{T}\left|\delta Z_{s}\right|^{2} \mathrm{~d} s\right] \leqslant & c^{\prime} \mathbb{E}_{t}\left[\Gamma_{T}|\delta \xi|^{2}+\int_{t}^{T} \Gamma_{s}\left|\left({ }^{1} f-{ }^{2} f\right)\left(s,{ }^{1} Y_{s},{ }^{1} Z_{s}\right)\right|^{2} \mathrm{~d} s\right. \\
& \left.+\int_{t}^{T} \Gamma_{s}\left(\left|\mathfrak{P}\left({ }^{2} Y_{s}\right)-{ }^{2} Y_{s}\right|+\left|\mathfrak{P}\left({ }^{1} Y_{s}\right)-{ }^{1} Y_{s}\right|\right)\left(\left|{ }^{1} \Phi_{s}\right|+\left|{ }^{2} \Phi_{s}\right|\right) \mathrm{d} s\right] .
\end{aligned}
$$

Proof. In this proof, we denote $A=\left(a^{i j}\right)=\left(H^{-1}\right)^{\dagger}$ and the following simplified notation will be used $a_{t}^{i j}=a^{i j}\left(t,{ }^{1} Y_{t}\right), \partial_{t} a_{t}^{i j}=\partial_{t} a^{i j}\left(t,{ }^{1} Y_{t}\right), \partial_{y} a_{t}^{i j}=\partial_{y} a{ }^{i j}\left(t,{ }^{1} Y_{t}\right), \partial_{y y}^{2} a_{t}^{i j}=$ $\partial_{y y}^{2} a^{i j}\left(t,{ }^{1} Y_{t}\right)$ and $f_{t}=f\left(t,{ }^{1} Y_{t},{ }^{1} Z_{t}\right)$. For the reader's convenience, we shall also denote $\sigma:=\sigma^{1 \xi} \vee \sigma^{2} \xi$ in the proof below.

1. We first show the integrability property of $\Gamma$. We first recall that from Proposition 2.1, for all $b>0$, we have

$$
\mathbb{E}\left[e^{b \int_{0}^{T}\left\{\left|\Theta_{s}^{\Phi}\right|+\left|\Theta_{s}^{f}\right|\right\} \mathrm{d} s}\right] \leqslant C_{K, \sigma, b} .
$$

Setting $p:=p(\Lambda)>1$ such that $p^{2} \mathfrak{B}(\Lambda) \leqslant \lambda_{\xi}$, recall Definition 2.1, we obtain using Hölder inequality,

$$
\begin{aligned}
\mathbb{E}\left[\left|\Gamma_{T}\right|^{p}\right] & \leqslant C_{\Lambda} \mathbb{E}\left[e^{p \mathfrak{B}(\Lambda) \int_{0}^{T}\left\{\Theta_{s}^{1}+\Theta_{s}^{\Phi}+\Theta_{s}^{f}\right\} \mathrm{d} s}\right] \\
& \leqslant C_{K, \Lambda, \sigma} \mathbb{E}\left[e^{p^{2} \mathfrak{B}(\Lambda) \int_{0}^{T} \Theta_{s}^{1} \mathrm{~d} s}\right]^{\frac{1}{p}} \\
& \leqslant C_{K, \Lambda, \sigma},
\end{aligned}
$$


where we used 2.30) and Proposition 2.1 (ii) .

2.a To obtain the stability result, we first expand the product $\left(\Gamma_{t} \delta Y_{t}^{\dagger} A\left(t, Y_{t}\right) \delta Y_{t}\right)_{0 \leqslant t \leqslant T}$. Applying Itô's formula, we compute, for $1 \leqslant i, j \leqslant d$,

$$
\begin{aligned}
& \mathrm{d}\left[\Gamma_{t} a_{t}^{i j} \delta Y_{t}^{i} \delta Y_{t}^{j}\right] / \Gamma_{t} \\
& =\delta Y_{t}^{i} \delta Y_{t}^{j}\left(a_{t}^{i j} \mathfrak{A}(\Lambda)+\partial_{t} a_{t}^{i j}\right) \mathrm{d} t=:\left(\mathcal{E}_{t}^{\mathcal{T}}\right)^{i j} \mathrm{~d} t \\
& +\delta Y_{t}^{i} \delta Y_{t}^{j}\left(a_{t}^{i j} \mathfrak{B}(\Lambda) \Theta_{t}^{1}+\frac{1}{2} \operatorname{Tr}\left[\partial_{y y}^{2} a_{t}^{i j 1} Z_{t}^{1} Z_{t}^{\dagger}\right]\right) \mathrm{d} t=:\left(\mathcal{E}_{t}^{Z}\right)^{i j} \mathrm{~d} t \\
& +\left\{a_{t}^{i j}\left(-\delta Y_{t}^{i} \delta f_{t}^{j}-\delta Y_{t}^{j} \delta f_{t}^{i}+\mathfrak{B}(\Lambda) \Theta_{t}^{f} \delta Y_{t}^{i} \delta Y_{t}^{j}\right)-\partial_{y} a_{t}^{i j 1} f_{t} \delta Y_{t}^{i} \delta Y_{t}^{j}\right\} \mathrm{d} t=:\left(\mathcal{E}_{t}^{f}\right)^{i j} \mathrm{~d} t \\
& +\left\{a_{t}^{i j} \sum_{m=1}^{k} \delta Z_{t}^{i m} \delta Z_{t}^{j m}+\sum_{m=1}^{k} \partial_{y} a_{t}^{i j} Z_{t}^{m}\left(\delta Y_{t}^{i} \delta Z_{t}^{j m}+\delta Y_{t}^{j} \delta Z_{t}^{i m}\right)\right\} \mathrm{d} t=:\left(\mathcal{E}_{t}^{\delta Z}\right)^{i j} \mathrm{~d} t \\
& +\left\{a_{t}^{i j}\left(\delta Y_{t}^{i} \delta Z_{t}^{j .}+\delta Y_{t} \delta Z_{t}^{i \cdot}\right)+\delta Y_{t}^{i} \delta Y_{t}^{j} \partial_{y} a_{t}^{i j 1} Z_{t}\right\} \mathrm{d} W_{t}=: \mathrm{d} M_{t}^{i j} \\
& +\left\{a_{t}^{i j}\left(\delta Y_{t}^{i} \delta \Psi_{t}^{j}+\delta Y_{t}^{j} \delta \Psi_{t}^{i}\right)+\left(\partial_{y} a_{t}^{i j 1} \Psi_{t}+\mathfrak{B}(\Lambda) a_{t}^{i j} \Theta_{t}^{\Phi}\right) \delta Y_{t}^{i} \delta Y_{t}^{j}\right\} \mathrm{d} t=:\left(\mathcal{E}_{t}^{\mathcal{R}}\right)^{i j} \mathrm{~d} t
\end{aligned}
$$

We now study each term separately.

2.b We start by the reflection terms in 2.35). We first observe that

$$
\sum_{1 \leqslant i, j \leqslant d}\left(\mathcal{E}_{t}^{\mathcal{R}}\right)^{i j}=A\left(t,{ }^{1} Y_{t}\right) \delta Y_{t} \cdot \delta \Psi_{t}+\sum_{1 \leqslant i, j \leqslant d} \partial_{y} a_{t}^{i j 1} \Psi_{t}+\mathfrak{B}(\Lambda) \Theta_{t}^{\Phi} \delta Y_{t} \cdot A\left(t,{ }^{1} Y_{t}\right) \delta Y_{t}
$$

Recalling (2.6) and (2.7), we compute

$$
\sum_{1 \leqslant i, j \leqslant d}\left(\mathcal{E}_{t}^{\mathcal{R}}\right)^{i j} \geqslant A\left(t,{ }^{1} Y_{t}\right) \delta Y_{t} \cdot \delta \Psi_{t}+\left(\frac{\mathfrak{B}(\Lambda)}{L} \Theta_{t}^{\Phi}-\left.C_{\Lambda}\right|^{1} \Phi_{t} \mid\right)\left|\delta Y_{t}\right|^{2} .
$$

For the first term in the right hand side of 2.36$)$, we compute $A\left(t,{ }^{1} Y_{t}\right) \delta Y_{t} \cdot \delta \Psi_{t}=A\left(t,{ }^{1} Y_{t}\right) \delta Y_{t} \cdot{ }^{1} \Psi_{t}-A\left(t,{ }^{2} Y_{t}\right) \delta Y_{t} \cdot{ }^{2} \Psi_{t}-\left\{A\left(t,{ }^{1} Y_{t}\right)-A\left(t,{ }^{2} Y_{t}\right)\right\} \delta Y_{t} \cdot{ }^{2} \Psi_{t}$.

We now observe that,

$$
\begin{aligned}
A\left(t,{ }^{1} Y_{t}\right) \delta Y_{t} \cdot{ }^{1} \Psi_{t} & =\delta Y_{t} \cdot A^{\dagger}\left(t,{ }^{1} Y_{t}\right) \Psi_{t}=\delta Y_{t} \cdot{ }^{1} \Phi_{t} \\
& \geqslant\left({ }^{1} Y_{t}-\mathfrak{P}\left({ }^{1} Y_{t}\right)+\mathfrak{P}\left({ }^{2} Y_{t}\right)-{ }^{2} Y_{t}\right) \cdot{ }^{1} \Phi_{t} \\
& \geqslant-\left(\left|\mathfrak{P}\left({ }^{1} Y_{t}\right)-{ }^{1} Y_{t}\right|+\left|\mathfrak{P}\left({ }^{2} Y_{t}\right)-{ }^{2} Y_{t}\right|\right)\left|{ }^{1} \Phi_{t}\right|
\end{aligned}
$$

where we used $(2.28)$ and the convexity property of $\mathcal{D}$. Similarly, we compute

$$
-A\left(t,{ }^{2} Y_{t}\right) \delta Y_{t} \cdot{ }^{2} \Psi_{t} \geqslant-\left(\left|\mathfrak{P}\left({ }^{1} Y_{t}\right)-{ }^{1} Y_{t}\right|+\left|\mathfrak{P}\left({ }^{2} Y_{t}\right)-{ }^{2} Y_{t}\right|\right)\left|{ }^{2} \Phi_{t}\right| .
$$

For the last term in the right-hand side of (2.37), we get, using the Lipschitz property of $A$ that

$$
\left\{A\left(t,{ }^{1} Y_{t}\right)-A\left(t,{ }^{2} Y_{t}\right)\right\} \delta Y_{t} \cdot{ }^{2} \Psi_{t} \geqslant-\left.C_{\Lambda}\left|\delta Y_{t}\right|^{2}\right|^{2} \Phi_{t} \mid
$$


Combining (2.38)-2.39)-2.40) with 2.36$)$, we obtain, for $\mathfrak{B}(\Lambda)$ large enough, $\mathbb{E}_{t}\left[\int_{t}^{T} \Gamma_{s} \sum_{1 \leqslant i, j \leqslant d}\left(\mathcal{E}_{s}^{\mathcal{R}}\right){ }^{i j} \mathrm{~d} s\right] \geqslant-C_{\Lambda} \mathbb{E}_{t}\left[\int_{t}^{T} \Gamma_{s}\left(\left|\mathfrak{P}\left({ }^{2} Y_{s}\right)-{ }^{2} Y_{s}\right|+\left|\mathfrak{P}\left({ }^{1} Y_{s}\right)-{ }^{1} Y_{s}\right|\right)\left(\left|{ }^{1} \Phi_{s}\right|+\left|{ }^{2} \Phi_{s}\right|\right) \mathrm{d} s\right]$.

2.c Using Young's inequality, we compute, recalling (2.6) and 2.7),

$$
\sum_{1 \leqslant i, j \leqslant d}\left(\mathcal{E}_{t}^{\delta Z}\right)^{i j} \geqslant \frac{1}{2 L}\left|\delta Z_{t}\right|^{2}-C_{\Lambda}\left|\delta Y_{t}\right|^{2}\left|Z_{t}\right|^{2}
$$

The terms $\mathcal{E}^{f}$ in 2.32 can be lower bounded, using Young's inequality and 2.6), by

$$
\begin{aligned}
\sum_{1 \leqslant i, j \leqslant d}\left(\mathcal{E}_{t}^{f}\right)^{i j} \geqslant & -\frac{1}{3 L}\left|\delta Z_{t}\right|^{2}+\left(\frac{\mathfrak{B}(\Lambda)}{L} \Theta_{t}^{f}-C_{K, \Lambda, \sigma}\left(1+\left|\theta_{t}{ }^{{ }^{\xi}}\right|+\left|{ }^{1} Z_{t}-\mathcal{Z}^{1} \xi\right|\right)\right)\left|\delta Y_{t}\right|^{2} \\
& -C_{\Lambda}\left|\left({ }^{1} f-{ }^{2} f\right)\left(t,{ }^{1} Y_{t},{ }^{1} Z_{t}\right)\right|^{2}
\end{aligned}
$$

recalling (2.19). We also have that

$$
\sum_{1 \leqslant i, j \leqslant d}\left(\mathcal{E}_{t}^{Z}\right)^{i j} \geqslant\left(\frac{\mathfrak{B}(\Lambda)}{L} \Theta_{t}^{1}-\left.\left.C_{\Lambda}\right|^{1} Z_{t}\right|^{2}\right)\left|\delta Y_{t}\right|^{2} \text { and } \sum_{1 \leqslant i, j \leqslant d}\left(\mathcal{E}_{t}^{\mathcal{T}}\right)^{i j} \geqslant\left(\frac{\mathfrak{A}(\Lambda)}{L}-C_{\Lambda}\right)\left|\delta Y_{t}\right|^{2} .
$$

2.d We now consider the local martingale $\mathcal{M}$, defined by

$$
\mathcal{M}_{t}:=\sum_{1 \leqslant i, j \leqslant d} \mathcal{M}_{t}^{i j} \text { with } \mathcal{M}_{t}^{i j}:=\int_{0}^{t} \Gamma_{s} \mathrm{~d} M_{s}^{i j}
$$

and are going to show that it is in fact a true martingale. We study only the second term in 2.34, the first term is treated similarly with $\delta Z$ in place of ${ }^{1} Z$. Applying Burkholder-Davis-Gundy inequality, we compute, using (2.7),

$$
\begin{aligned}
\mathbb{E}\left[\sup _{t \in[0, T]}\left|\int_{0}^{t} \Gamma_{t} \delta Y_{t}^{i} \delta Y_{t}^{j} \partial_{y} a_{t}^{i j 1} Z_{t} \mathrm{~d} W_{t}\right|\right] & \leqslant C_{\Lambda} \mathbb{E}\left[\left(\int_{0}^{T} \Gamma_{t}^{2}\left|\delta Y_{t}\right|^{2}\left|{ }^{1} Z_{t}\right|^{2} \mathrm{~d} t\right)^{\frac{1}{2}}\right] \\
& \leqslant C_{\Lambda, p} \mathbb{E}\left[\left|\Gamma_{T}\right|^{p}\right]^{\frac{1}{p}} \mathbb{E}\left[\sup _{t \in[0, T]}\left|\delta Y_{t}\right|^{q}\left(\int_{0}^{T}\left|Z_{t}\right|^{2} \mathrm{~d} t\right)^{q}\right]^{\frac{1}{q}} \\
& \leqslant C_{\Lambda, p} \mathbb{E}\left[\left|\Gamma_{T}\right|^{p}\right]^{\frac{1}{p}}
\end{aligned}
$$

where we used Cauchy-Schwarz inequality, the energy inequality, with the fact that $\sup _{t}\left|\delta Y_{t}\right|$ is bounded in any $\mathscr{L}^{r}\left(\mathcal{F}_{T}\right)$ and ${ }^{1} Z \in \mathscr{B}^{2}$. From step 1 . we deduce then that 
the supremum of the local martingale term is integrable and it is thus a martingale.

3. Combining the results from steps 2.a - 2.c, we get, for $\mathfrak{B}(\Lambda)$ and $\mathfrak{A}(\Lambda)$ large enough,

$$
\begin{gathered}
\Gamma_{t} \delta Y_{t} \cdot A\left(t, Y_{t}\right) \delta Y_{t}+\mathbb{E}_{t}\left[\int_{t}^{T} \Gamma_{s} \frac{1}{6 L}\left|\delta Z_{s}\right|^{2} \mathrm{~d} s+\mathcal{M}_{t}-\mathcal{M}_{T}\right] \\
\leqslant \\
\mathbb{E}_{t}\left[\Gamma_{T} \delta \xi \cdot A\left(T, Y_{T}\right) \delta \xi\right]+ \\
C_{\Lambda, K, \sigma} \mathbb{E}_{t}\left[\int_{t}^{T} \Gamma_{s}\left\{\left|\left({ }^{1} f-{ }^{2} f\right)\left(s,{ }^{1} Y_{s},{ }^{1} Z_{s}\right)\right|^{2}+\left(\left|\mathfrak{P}\left({ }^{2} Y_{s}\right)-{ }^{2} Y_{s}\right|+\left|\mathfrak{P}\left({ }^{1} Y_{s}\right)-{ }^{1} Y_{s}\right|\right)\left(\left|{ }^{1} \Phi_{s}\right|+\left|{ }^{2} \Phi_{s}\right|\right)\right\} \mathrm{d} s\right] .
\end{gathered}
$$

Step 2.d allows us to claim that $\mathbb{E}_{t}\left[\mathcal{M}_{t}-\mathcal{M}_{T}\right]=0$ in the previous inequality. Moreover, we have, recalling (2.6) and $\Gamma \geqslant 1$,

$$
\left|\delta Y_{t}\right|^{2} \leqslant L \Gamma_{t} \delta Y_{t} \cdot A\left(t, Y_{t}\right) \delta Y_{t} \text { and } \mathbb{E}_{t}\left[\Gamma_{T} \delta \xi \cdot A\left(T, Y_{T}\right) \delta \xi\right] \leqslant L \mathbb{E}_{t}\left[\Gamma_{T}|\delta \xi|^{2}\right],
$$

which combined with the previous inequality concludes the proof of (2.29).

Remark 2.6. The dependence upon $\Lambda$ is a key fact that will restrain us to extend straightforwardly to rougher coefficients our main existence and uniqueness results in the non-Markovian case, recall assumption (SB). This is a quite important limitation: indeed, when we consider classical applications of obliquely reflected BSDEs to optimal switching problems we have to deal with convex polytopes domains $\mathcal{D}$ which are corner domains. Thus, in this context $H$ is not smooth enough to apply our results. It justifies Section 4 where the Markovian framework is studied under a weaker regularity assumption on $H$ thanks to a different approach.

\subsection{Some interesting facts about the class $\mathfrak{T}_{\beta}$}

We first make the following observation.

Proposition 2.3. Let $\xi \in \mathscr{L}^{2}\left(\mathcal{F}_{T}\right)$ satisfying $(\boldsymbol{S B})(i)$. If we have, for some $\beta>0$,

$$
d_{\mathscr{B}^{2}}\left(\mathcal{Z}^{\xi}, \mathscr{H}^{\infty}\right)<\frac{1}{\sqrt{\beta}},
$$

then $\xi \in \mathfrak{T}_{\beta}$.

Proof. We can find $V \in \mathscr{H}^{\infty}$, s.t. $\left\|\mathcal{Z}^{\xi}-V\right\|_{\mathscr{B}^{2}}=\frac{1}{(1+\eta) \sqrt{\beta}}$, for some $\eta>0$ small enough. We now set $\lambda:=\left(\frac{1+\frac{\eta}{2}}{1+\frac{\eta}{3}}\right)^{2} \beta$ and we compute, using Young's inequality,

$$
\left|\mathcal{Z}^{\xi}\right|^{2} \leqslant\left(1+\frac{\eta}{3}\right)\left|\mathcal{Z}^{\xi}-V\right|^{2}+\left(1+\frac{3}{\eta}\right)|V|^{2} .
$$

This leads, using Hölder inequality, to

$$
\mathbb{E}\left[e^{\lambda \int_{0}^{T}\left|\mathcal{Z}_{t}^{\xi}\right|^{2} \mathrm{~d} t}\right] \leqslant C \mathbb{E}\left[e^{\left(1+\frac{\eta}{3}\right)^{2} \lambda \int_{0}^{T}\left|\mathcal{Z}_{t}^{\xi}-V_{t}\right|^{2} \mathrm{~d} t}\right]^{\frac{1}{1+\frac{\eta}{3}}}
$$


where we used the fact that $V \in \mathscr{H}^{\infty}$. Since $\left\|\left(1+\frac{\eta}{3}\right) \sqrt{\lambda}\left(\mathcal{Z}^{\xi}-V\right)\right\|_{\mathscr{B}^{2}}=\frac{1+\frac{\eta}{2}}{1+\eta}<1$, we can apply the John-Nirenberg inequality, see Theorem 2.2 in [14], to obtain

$$
\mathbb{E}\left[e^{\left(1+\frac{\eta}{3}\right)^{2} \lambda \int_{0}^{T}\left|\mathcal{Z}_{t}^{\xi}-V_{t}\right|^{2} \mathrm{~d} t}\right]<\infty,
$$

which concludes the proof.

Proposition 2.3 only suggests a sufficient condition. In the case $\beta=+\infty$, for which condition $(2.45)$ should read $d_{\mathscr{B}^{2}}\left(\mathcal{Z}^{\xi}, \mathscr{H}^{\infty}\right)=0$, it is known that the condition is not necessary. We refer the interested reader to the paper [23], where this question is treated with more details.

The next result shows that a class of path-dependent function of some smooth processes are naturally contained in $\mathfrak{T}_{\beta}$ and actually for all $\beta>0$. This class is quite important for applications.

Proposition 2.4. Let $X \in \mathscr{S}^{2}$ such that for all $t, s \leqslant T$, the Malliavin derivatives of $X_{s}$ denoted $D_{t} X_{s}$ is well defined and satisfies $\left\|\sup _{t, s}\left|\mathbb{E}_{t}\left[D_{t} X_{s}\right]\right|\right\|_{\mathscr{L}_{\infty}}<\infty$. Let $g: \mathcal{C}^{0}\left([0, T], \mathbb{R}^{n}\right) \rightarrow \mathbb{R}^{d}$ be a uniformly continuous function, then denoting $\xi=$ $g\left(\left(X_{s}\right)_{s \in[0, T]}\right)$, we have that $\mathcal{Z}^{\xi} \in \overline{\mathscr{H}}^{\infty} \mathscr{B}^{2}$.

1.a We first start by considering a sequence $\left(g_{N}\right)$ of $N$-Lipschitz regularisation of $g=\left(g^{1}, \ldots, g^{d}\right)$ given by

$$
g_{N}^{i}(x)=\inf _{u \in \mathcal{C}^{0}\left([0, T], \mathbb{R}^{n}\right)}\left\{g^{i}(u)+N\|u-x\|_{\infty}\right\}, \quad \text { for all } x \in \mathcal{C}^{0}\left([0, T], \mathbb{R}^{n}\right), \quad 1 \leqslant i \leqslant n .
$$

Let us observe that $g_{N}$ is finite for $N$ large enough due to the linear growth of $g$. Then we have, for all $x \in \mathcal{C}^{0}\left([0, T], \mathbb{R}^{n}\right)$ and $1 \leqslant i \leqslant n$,

$$
\begin{aligned}
g^{i}(x) \geqslant g_{N}^{i}(x) & \geqslant \inf _{u \in \mathcal{C}^{0}\left([0, T], \mathbb{R}^{n}\right)}\left\{g^{i}(x)-\omega_{g^{i}}\left(|u-x|_{\infty}\right)+N|u-x|_{\infty}\right\} \\
& \geqslant g^{i}(x)+\inf _{u \in \mathcal{C}^{0}\left([0, T], \mathbb{R}^{n}\right)}\left\{N|u|_{\infty}-\omega_{g^{i}}\left(|u|_{\infty}\right)\right\}
\end{aligned}
$$

where $\omega_{g^{i}}$ is a concave modulus of continuity for the uniformly continuous component $g^{i}$ of $g$. Thus we get

$$
\left|g_{N}-g\right|_{\infty} \leqslant C \sum_{i=1}^{d} \sup _{u \in \mathcal{C}^{0}\left([0, T], \mathbb{R}^{n}\right)}\left\{\omega_{g^{i}}\left(|u|_{\infty}\right)-N|u|_{\infty}\right\}:=c(N) .
$$

Since $\omega_{g^{i}}(h)=o(1)$ when $h \rightarrow 0^{+}$then $c(N)=o(1)$ when $N \rightarrow+\infty$.

1.b Defining $\mathcal{Y}_{t}^{N}:=\mathbb{E}_{t}\left[g_{N}(X).\right]=g_{N}(X)-.\int_{t}^{T} \mathcal{Z}_{s}^{N} \mathrm{~d} W_{s}$ and applying Itô's formula to $\left|\mathcal{Y}^{N}-\mathcal{Y}_{\cdot}^{\xi}\right|^{2}$, we compute

$$
\left|Y_{t}^{N}-\mathcal{Y}_{t}^{\xi}\right|^{2}+\mathbb{E}_{t}\left[\int_{t}^{T}\left|\mathcal{Z}_{t}^{N}-\mathcal{Z}_{t}^{\xi}\right|^{2} \mathrm{~d} t\right]=\mathbb{E}_{t}\left[\left|g_{N}(X .)-g(X .)\right|^{2}\right] \leqslant c(N)^{2}
$$


recall (2.46). From this, we deduce that for all $\epsilon>0$, there exists $N_{\epsilon}$, s.t. for all $N \geqslant N_{\epsilon}$,

$$
\left\|\mathcal{Z}^{N}-\mathcal{Z}^{\xi}\right\|_{\mathscr{B}^{2}} \leqslant \epsilon
$$

2. We now show that $\mathcal{Z}^{N}$ introduced above, belongs to $\mathscr{H}^{\infty}$. This fact combined with 2.47) proves the statement of the proposition.

Following Lemma 4.1 in [16] there exists a family $\Pi=\{\pi\}$ of partitions of $[0, T]$ and a family of discrete functionals $\left\{g_{N, \pi}\right\}$ such that

- for each $\pi \in \Pi$, with $\pi: 0=t_{0}<\ldots<t_{m}=T$, we have that $g_{N, \pi} \in C_{b}^{\infty}\left(\mathbb{R}^{d(m+1)}\right)$, and satisfies

$$
\sum_{i=0}^{m}\left|\partial_{x_{i}} g_{N, \pi}(x)\right| \leqslant N, \quad \forall x \in \mathcal{C}^{0}\left([0, T], \mathbb{R}^{n}\right),
$$

where $g_{N, \pi}(x):=g_{N, \pi}\left(x\left(t_{0}\right), \ldots, x\left(t_{m}\right)\right)$.

- for any $x \in \mathcal{C}^{0}\left([0, T], \mathbb{R}^{n}\right)$ it holds that

$$
\lim _{|\pi| \rightarrow 0}\left|g_{N, \pi}(x)-g_{N}(x)\right|=0 .
$$

We naturally consider $\left(\mathcal{Y}^{N, \pi}, \mathcal{Z}^{N, \pi}\right)$ given by

$$
\mathcal{Y}_{t}^{N, \pi}:=\mathbb{E}_{t}\left[g_{N, \pi}(X)\right]=g_{N, \pi}(X)-\int_{t}^{T} \mathcal{Z}_{s}^{N, \pi} \mathrm{d} W_{s}, \quad 0 \leqslant t \leqslant T .
$$

2.a By the Clark-Ocone formula, we have that

$$
\begin{aligned}
\mathcal{Z}_{t}^{N, \pi} & =\mathbb{E}_{t}\left[D_{t} g_{N, \pi}(X)\right] \\
& =\sum_{i=1}^{m} \partial_{x_{i}} g_{N, \pi}(X) \mathbb{E}_{t}\left[D_{t} X_{t_{i}}\right] .
\end{aligned}
$$

Now, using (2.48) and the assumption on $\left(D_{t} X_{s}\right)_{t, s \leqslant T}$, we obtain

$$
\left\|\mathcal{Z}^{N, \pi}\right\|_{\mathscr{S}_{\infty}} \leqslant C_{N}
$$

2.b Combining (2.49) and the fact that $\left|g_{N}(X)-g_{N, \pi}(X)\right| \leqslant C_{N}\left(1+\sup _{t \in[0, T]}\left|X_{t}\right|\right) \in L^{2}$, we can use the dominated convergence theorem to get

$$
\lim _{|\pi| \rightarrow 0} \mathbb{E}\left[\left|g_{N}(X)-g_{N, \pi}(X)\right|^{2}\right]=0
$$

which leads to

$$
\lim _{|\pi| \rightarrow 0} \mathbb{E}\left[\int_{0}^{T}\left|\mathcal{Z}^{N}-\mathcal{Z}^{N, \pi}\right|^{2} \mathrm{~d} t\right]=0
$$


Up to a subsequence, we have $\mathcal{Z}^{N, \pi} \rightarrow \mathcal{Z}^{N} \mathrm{~d} \mathbb{P} \otimes \mathrm{d} t$-a.e. and, moreover, $\left|\mathcal{Z}^{N, \pi}\right| \leqslant C_{N}$, recall (2.50). We thus obtain for (a version of) the limit process

$$
\int_{0}^{T}\left|\mathcal{Z}_{t}^{N}\right|^{2} \mathrm{~d} t \leqslant T C_{N}^{2}, \quad \mathbb{P}-a . s .
$$

which concludes the proof of this step.

3. Finally, we remark that $\mathcal{Z}^{\xi} \in \mathscr{B}^{2}$ since $\mathcal{Z}^{\xi}-\mathcal{Z}^{N} \in \mathscr{B}^{2}$ and $\mathcal{Z}^{N} \in \mathscr{H}^{\infty} \subset \mathscr{B}^{2}$. We conclude the proof by using (2.47).

We obtain the following direct corollary, which gives a sufficient condition on models in a path-dependent framework to check the admissibility of the terminal condition.

Corollary 2.2. Let $X$ be solution of the Lipschitz SDE

$$
X_{t}=x+\int_{0}^{t} b\left(X_{s}\right) d s+\int_{0}^{t} \sigma\left(X_{s}\right) d W_{s}
$$

where $\sigma$ and $b$ are Lipschitz continuous functions and $\sigma$ is bounded.

Set $\xi:=g\left(\left(X_{s}\right)_{t \in[0, T]}\right)$ where $g$ is a uniformly continuous function on $\mathcal{C}\left([0, T], \mathbb{R}^{d}\right)$ with linear growth, then $\xi$ belongs to $\mathfrak{T}_{\beta}$, for all $\beta>0$. Moreover, if $\tilde{\xi} \in \mathscr{L}^{\infty}$, then $\xi+\tilde{\xi}$ belongs to $\mathfrak{T}_{\beta}$ for all $\beta<\|\tilde{\xi}\|_{\mathscr{L} \infty}^{-2}$.

Proof. When $\sigma$ and $b$ are smooth enough, it is well known, see e.g. 18, that $X$ is Malliavin differentiable and, for all $1 \leqslant i \leqslant k,\left(D_{t}^{i} X_{s}\right)_{s \in[t, T]}$ is solution of the linear SDE given by

$$
D_{t}^{i} X_{s}=\sigma^{i}\left(X_{t}\right)+\int_{t}^{s} \nabla b\left(X_{r}\right) D_{t}^{i} X_{r} \mathrm{~d} r+\int_{t}^{s} \sum_{j=1}^{k} \nabla \sigma^{j}\left(X_{r}\right) D_{t}^{i} X_{r} \mathrm{~d} W_{r}^{j}, \quad t \leqslant s \leqslant T .
$$

Then, we easily get that $\left|\mathbb{E}_{t}\left[D_{t} X_{s}\right]\right| \leqslant e^{K_{b} T} M$ with $K_{b}$ the Lipschitz constant of $b$ and $M$ a bound of $\sigma$. Thus we can apply Proposition 2.4 to get the first part of the result. When coefficients are not smooth enough, a standard approximation gives us the result, pointing out the fact that $\left\|\sup _{t, s}\left|\mathbb{E}_{t[}\left[D_{t} X_{s}\right]\right|\right\|_{\mathscr{L}_{\infty}}$ can be uniformly bounded with respect to the approximation. For the second part of the corollary, we just have to remark that

$$
d_{\mathscr{B}^{2}}\left(\mathcal{Z}^{\xi+\tilde{\xi}}, \mathscr{H}^{\infty}\right) \leqslant\left\|\mathcal{Z}^{\xi+\tilde{\xi}}-\mathcal{Z}^{\xi}\right\|_{\mathscr{B}^{2}}+d_{\mathscr{B}^{2}}\left(\mathcal{Z}^{\xi}, \mathscr{H}^{\infty}\right)=\left\|\mathcal{Z}^{\tilde{\xi}}\right\|_{\mathscr{B}^{2}} .
$$

Moreover, applying Itô's formula to $\left|\mathcal{Y}_{t}^{\tilde{\xi}}\right|^{2}$, we compute

$$
\left\|\mathcal{Z}^{\tilde{\xi}}\right\|_{\mathscr{B}^{2}} \leqslant\|\tilde{\xi}\|_{\mathscr{L}^{\infty}}
$$

which implies

$$
d_{\mathscr{B}^{2}}\left(\mathcal{Z}^{\xi+\tilde{\xi}}, \mathscr{H}^{\infty}\right) \leqslant\|\tilde{\xi}\|_{\mathscr{L}^{\infty}} .
$$

Thus, we just have to apply Proposition 2.3 to conclude. 


\section{$3 \quad$ Existence and uniqueness in a regular setting}

In this section, we obtain an existence and uniqueness result in a non Markovian setting, working under assumption (SB) and considering terminal condition in the class $\mathfrak{T}_{\beta}$, for some $\beta>0$. This $\beta$, as shown in the previous section depends dramatically on the smoothness of the coefficients. Our proof is done in two main steps. In the first step, we restrict to the case of a bounded terminal condition. We study the wellposedness of the penalised equation, and prove their convergence to an obliquely reflected BSDE. In a second step, we extend our result to all terminal condition in the class $\mathfrak{T}_{\beta}$.

\subsection{Bounded terminal condition}

We first obtain some results on the penalised BSDE that will be used later in this section and also in Section 4 in the Markovian case. We thus essentially work here under the assumption (A).

We start with the following lemma that verifies the well-posedness of equation 1.2 under some classical conditions.

Lemma 3.1. We assume that $(\boldsymbol{A})$ is in force and that $f$ and $H$ are Lipschitz continuous with respect to $(y, z)$. Then, for all $n \in \mathbb{N}$ there exists a unique solution to (1.2) in $\mathscr{S}^{2} \times \mathscr{H}^{2}$.

Proof. Since $\mathcal{D}$ is convex, $\varphi_{n}^{M}$ is convex and $n M$-Lipschitz continuous, recall (1.3). Indeed, denoting $\mathcal{D}_{M}:=\left\{y \in \mathbb{R}^{d} \mid d(y, \mathcal{D}) \leqslant M\right\}$, we have that

$$
\varphi_{n}^{M}(h)=\left\{\begin{array}{lll}
n \frac{1}{2} d^{2}(h, \mathcal{D}) & \text { if } & h \in \mathcal{D}_{M} \\
n M d(h, \mathcal{D})-\frac{n M^{2}}{2} & \text { if } & h \notin \mathcal{D}_{M}
\end{array}\right.
$$

and

$$
\nabla \varphi_{n}^{M}(y)=\left\{\begin{array}{lll}
0 & \text { if } \quad y \in \overline{\mathcal{D}} \\
n d(y, \mathcal{D}) \frac{y-\mathfrak{P}(y)}{|y-\mathfrak{P}(y)|} & \text { if } \quad y \in \mathcal{D}_{M} \backslash \overline{\mathcal{D}} \\
n M \frac{y-\mathfrak{P}(y)}{|y-\mathfrak{P}(y)|} & \text { if } \quad y \notin \mathcal{D}_{M}
\end{array}\right.
$$

Finally $H$ and $\nabla \varphi_{n}^{M}$ are two Lipschitz bounded functions which proves that the penalised BSDE (1.2) has a Lipschitz driver: the classical result of [19] then applies to get the existence and uniqueness result.

Lemma 3.2. Assume that $(\boldsymbol{A})$ holds and that there exists a solution to 1.2 in $\mathscr{S}^{2} \times$ $\mathscr{H}^{2}$. Then, $\left(Y^{n}, Z^{n}, \nabla \varphi_{n}^{M}\left(Y^{n}\right)\right)$ satisfies Condition (2.12) for some $K>0$ and for some $c>0$ we have

$$
\sup _{t \in[0, T]} \mathbb{E}\left[\varphi_{n}^{M}\left(Y_{t}^{n}\right)\right]+\mathbb{E}\left[\int_{0}^{T}\left|\nabla \varphi_{n}^{M}\left(Y_{s}^{n}\right)\right|^{2} \mathrm{~d} s\right] \leqslant c \mathbb{E}\left[|\xi|^{2}+\int_{0}^{T}\left|\alpha_{s}\right|^{2} d s\right] .
$$


Importantly, $K$ and $c$ do not depend on $n$, nor $M$.

Moreover, if (SB) holds, then, there exists $c^{\prime}:=c^{\prime}\left(\sigma^{\xi}\right)$, which does not depend on $n$ nor $M$, such that

$$
\sup _{t \in[0, T]} \varphi_{n}^{M}\left(Y_{t}^{n}\right)+\left\|\nabla \varphi_{n}^{M}\left(Y^{n}\right)\right\|_{\mathscr{B}^{2}}^{2} \leqslant c^{\prime} .
$$

Proof. Since $\varphi_{n}^{M}$ is a $C^{1}$ convex function, we have the following inequality (see Lemma 2.38 in [20]): for $s \in[t, T]$,

$$
\begin{aligned}
& \varphi_{n}^{M}\left(Y_{s}^{n}\right)+\int_{s}^{T} \nabla \varphi_{n}^{M}\left(Y_{u}^{n}\right) \cdot H\left(u, Y_{u}^{n}, Z_{u}^{n}\right) \nabla \varphi_{n}^{M}\left(Y_{u}^{n}\right) \mathrm{d} u \\
& \quad \leqslant \varphi_{n}^{M}(\xi)+\int_{s}^{T} \nabla \varphi_{n}^{M}\left(Y_{u}^{n}\right) \cdot f\left(u, Y_{u}^{n}, Z_{u}^{n}\right) \mathrm{d} u-\int_{s}^{T} \nabla \varphi_{n}^{M}\left(Y_{u}^{n}\right) \cdot Z_{u}^{n} \mathrm{~d} W_{u},
\end{aligned}
$$

and we recall that $\varphi_{n}^{M}(\xi)=0$. We observe, using (2.2) that

$$
\nabla \varphi_{n}^{M}\left(Y_{u}^{n}\right) \cdot H\left(u, Y_{u}^{n}, Z_{u}^{n}\right) \nabla \varphi_{n}^{M}\left(Y_{u}^{n}\right) \geqslant \eta\left|\nabla \varphi_{n}^{M}\left(Y_{u}^{n}\right)\right|^{2}
$$

and combining Cauchy-Schwarz inequality with Young's inequality

$$
\int_{s}^{T} \nabla \varphi_{n}^{M}\left(Y_{u}^{n}\right) \cdot f\left(u, Y_{u}^{n}, Z_{u}^{n}\right) \mathrm{d} u \leqslant \frac{\eta}{2} \int_{s}^{T}\left|\nabla \varphi_{n}^{M}\left(Y_{u}^{n}\right)\right|^{2} \mathrm{~d} u+\frac{2}{\eta} \int_{s}^{T}\left|f\left(u, Y_{u}^{n}, Z_{u}^{n}\right)\right|^{2} \mathrm{~d} u .
$$

From this, we deduce

$$
\varphi_{n}^{M}\left(Y_{t}^{n}\right)+\mathbb{E}_{t}\left[\int_{t}^{T}\left|\nabla \varphi_{n}^{M}\left(Y_{u}^{n}\right)\right|^{2} \mathrm{~d} u\right] \leqslant \frac{4}{\eta} \mathbb{E}_{t}\left[\int_{t}^{T}\left|f\left(u, Y_{u}^{n}, Z_{u}^{n}\right)\right|^{2} \mathrm{~d} u\right],
$$

which proves (2.12) for $\left(Y^{n}, Z^{n}, \Phi^{n}\right)$. This allows then to invoke Lemma 2.1 to obtain (3.3) under (A). Under (SB), (3.7) allows also to conclude recalling that $f$ is Lipschitz continuous, $\theta^{\xi} \in \mathscr{B}^{2}$ and $(2.19)$.

We now prove our first existence result for the obliquely reflected BSDE

$$
\left\{\begin{array}{l}
Y_{t}=\xi+\int_{t}^{T} f\left(s, Y_{s}, Z_{s}\right) \mathrm{d} s-\int_{t}^{T} H\left(s, Y_{s}\right) \Phi_{s} \mathrm{~d} s-\int_{t}^{T} Z_{s} \mathrm{~d} W_{s}, \quad 0 \leqslant t \leqslant T, \\
Y_{t} \in \overline{\mathcal{D}}, \quad \Phi_{t} \in \partial \varphi\left(Y_{t}\right), \quad \int_{0}^{T} \mathbf{1}_{\left\{Y_{t} \notin \partial \mathcal{D}\right\}}\left|\Phi_{t}\right| \mathrm{d} t=0 .
\end{array}\right.
$$

Proposition 3.1. Assume that (SB) holds and that $\xi \in \mathscr{L}^{\infty} \cap \mathfrak{T}_{\mathfrak{B}(\Lambda)}$. Then, there exists a solution in $\mathscr{S}^{2} \times \mathscr{H}^{2} \times \mathscr{H}^{2}$ to the obliquely reflected BSDE (3.8).

Proof. To obtain the existence result, we consider a sequence of penalised BSDEs given by equation 1.2 for which we have existence and uniqueness from Lemma 3.1 . In the definition of $\varphi_{n}^{M}$, recall 1.3 , we set $M=2 c$ where $c$ is given in Corollary 2.1. In particular, we observe that for this choice of $M$, for $0 \leqslant t \leqslant T$,

$$
\Phi_{t}^{n}:=\nabla \varphi_{n}^{M}\left(Y_{t}^{n}\right)=n\left(Y_{t}^{n}-\mathfrak{P}\left(Y_{t}^{n}\right)\right) \text { and } \frac{1}{n} \varphi_{n}\left(Y_{t}^{n}\right)=\frac{1}{2}\left|\mathfrak{P}\left(Y_{t}^{n}\right)-Y_{t}^{n}\right|^{2},
$$


recall (3.1) and (3.2). We will use this fact later on.

1.a. We now prove that $\left(Y^{n}, Z^{n}\right)$ is a Cauchy sequence in $\mathscr{S}^{2} \times \mathscr{H}^{2}$. Indeed, let $m \geqslant 0$ and $n \geqslant 0$, thanks to Lemma 3.2 we can apply Proposition 2.2 to obtain

$$
\begin{aligned}
& \sup _{t \in[0, T]} \mathbb{E}\left[\left|Y_{t}^{n}-Y_{t}^{m}\right|^{2}\right]+\left\|Z^{n}-Z^{m}\right\|_{\mathscr{H}^{2}}^{2} \\
& \leqslant C_{\Lambda} \mathbb{E}\left[\int_{0}^{T} \Gamma_{s}^{n, m}\left(\left|\mathfrak{P}\left(Y_{s}^{n}\right)-Y_{s}^{n}\right|+\left|\mathfrak{P}\left(Y_{s}^{m}\right)-Y_{s}^{m}\right|\right)\left(\left|\Phi_{s}^{m}\right|+\left|\Phi_{s}^{n}\right|\right) \mathrm{d} s .\right]=: A^{n, m} .
\end{aligned}
$$

Let us notice that, from Proposition 2.2 again, there exist $p>1$ and a constant $C$ such that

$$
\mathbb{E}\left[\left|\Gamma_{T}^{n, m}\right|^{p}\right] \leqslant C,
$$

where, importantly, $p$ and $C$ do not depend on $(n, m)$. Applying Itô's formula to $\mid Y^{n}-$ $\left.Y^{m}\right|^{2}$ on $[0, T]$, we compute, using usual arguments,

$$
\begin{aligned}
\left\|Y^{n}-Y^{m}\right\|_{\mathscr{S}^{2}}^{2} & \leqslant C \mathbb{E}\left[\int_{0}^{T}\left|Y_{t}^{n}-Y_{t}^{m}\right|\left(\left|\Phi_{t}^{n}\right|+\left|\Phi_{t}^{m}\right|\right) \mathrm{d} t\right] \\
& +C \mathbb{E}\left[\sup _{t \in[0, T]}\left|\int_{0}^{t}\left(Y_{s}^{n}-Y_{s}^{m}\right)\left(Z_{s}^{n}-Z_{s}^{m}\right) \mathrm{d} W_{s}\right|\right] .
\end{aligned}
$$

Using Burkholder-Davis-Gundy inequality and Young's inequality, we obtain

$$
\left\|Y^{n}-Y^{m}\right\|_{\mathscr{S}^{2}}^{2} \leqslant C \mathbb{E}\left[\int_{0}^{T}\left|Y_{t}^{n}-Y_{t}^{m}\right|\left(\left|\Phi^{n}\right|+\left|\Phi_{t}^{m}\right|\right) \mathrm{d} t\right]+C\left\|Z^{n}-Z^{m}\right\|_{\mathscr{H}^{2}}^{2} .
$$

Applying Cauchy-Schwarz inequality, and using Lemma 3.2, we get

$$
\left\|Y^{n}-Y^{m}\right\|_{\mathscr{S}^{2}}^{2} \leqslant C\left(\left\|Y^{n}-Y^{m}\right\|_{\mathscr{H}^{2}}+\left\|Z^{n}-Z^{m}\right\|_{\mathscr{H}^{2}}^{2}\right) .
$$

Combining the previous inequality with 3.10$)$, we have

$$
\left\|Y^{n}-Y^{m}\right\|_{\mathscr{S}^{2}}^{2}+\left\|Z^{n}-Z^{m}\right\|_{\mathscr{H}^{2}}^{2} \leqslant C\left(A_{n, m}+\sqrt{A_{n, m}}\right) .
$$

1.b We now study the $A_{n, m}$ term. We first observe, recalling Lemma 3.2 and 3.9 ,

$$
\begin{aligned}
\mathbb{E}\left[\int_{0}^{T} \Gamma_{t}^{n, m}\left|\mathfrak{P}\left(Y_{t}^{n}\right)-Y_{t}^{n}\right|\left|\Phi_{s}^{m}\right| \mathrm{d} s\right] & \leqslant\left\|\sup _{t}\left|\mathfrak{P}\left(Y_{t}^{n}\right)-Y_{t}^{n}\right|\right\|_{\mathscr{L} \infty} \mathbb{E}\left[\Gamma_{T}^{n, m} \int_{0}^{T}\left|\Phi_{s}^{m}\right| \mathrm{d} s\right] \\
& \leqslant \frac{C}{\sqrt{n}} \mathbb{E}\left[\Gamma_{T}^{n, m} \int_{0}^{T}\left|\Phi_{s}^{m}\right| \mathrm{d} s\right] .
\end{aligned}
$$

Applying Hölder inequality, denoting $q$ the conjugate exponent of $p$ introduced in 3.11, we deduce from the previous inequality

$$
\mathbb{E}\left[\int_{0}^{T} \Gamma_{t}^{n, m}\left|\mathfrak{P}\left(Y_{t}^{n}\right)-Y_{t}^{n}\right|\left|\Phi_{s}^{m}\right| \mathrm{d} s\right] \leqslant \frac{C}{\sqrt{n}} \mathbb{E}\left[\left(\int_{0}^{T}\left|\Phi_{s}^{m}\right|^{2} \mathrm{~d} s\right)^{\frac{q}{2}}\right] .
$$


Then, combining the energy inequality with (3.4), we conclude

$$
\mathbb{E}\left[\int_{0}^{T} \Gamma_{t}^{n, m}\left|\mathfrak{P}\left(Y_{t}^{n}\right)-Y_{t}^{n}\right|\left|\Phi_{s}^{m}\right| \mathrm{d} s\right] \leqslant \frac{C}{\sqrt{n}} .
$$

Similarly we obtain,

$$
\mathbb{E}\left[\int_{0}^{T} \Gamma_{t}^{n, m}\left|\mathfrak{P}\left(Y_{t}^{m}\right)-Y_{t}^{m}\right|\left|\Phi_{s}^{n}\right| \mathrm{d} s\right] \leqslant \frac{C}{\sqrt{m}}
$$

and

$$
\mathbb{E}\left[\int_{0}^{T} \Gamma_{t}^{n, m}\left(\left|\mathfrak{P}\left(Y_{t}^{n}\right)-Y_{t}^{n}\right|+\left|\mathfrak{P}\left(Y_{t}^{m}\right)-Y_{t}^{m}\right|\right)\left|\Phi_{s}^{m}\right| \mathrm{d} s\right] \leqslant C\left(\frac{1}{\sqrt{m}}+\frac{1}{\sqrt{n}}\right) .
$$

Combining the previous inequalities with $(3.13)$, we compute that

$$
\left\|Y^{n}-Y^{m}\right\|_{\mathscr{S}^{2}}^{2}+\left\|Z^{n}-Z^{m}\right\|_{\mathscr{H}^{2}}^{2} \leqslant C\left(n^{-\frac{1}{4}}+m^{-\frac{1}{4}}\right),
$$

which proves that $\left(Y^{n}, Z^{n}\right)_{n}$ is a Cauchy sequence in $\mathscr{S}^{2} \times \mathscr{H}^{2}$. We denote $(Y, Z)$ its limit.

2. We now prove that $(Y, Z)$ is solution to an obliquely reflected BSDE, namely we pass to the limit in $(1.2)$. Let us first observe that, passing to the limit in (3.4) yields that $Y \in \overline{\mathcal{D}}$ as expected.

2.a We first study the reflecting term. Since, by Lemma 3.2 .

$$
\mathbb{E}\left[\int_{0}^{T}\left|\nabla \varphi_{n}\left(Y_{s}^{n}\right)\right|^{2} \mathrm{~d} s\right] \leqslant C
$$

we have, up to a subsequence, the following weak $L^{2}([0, T] \times \Omega)$-convergence:

$$
\nabla \varphi_{n}\left(Y^{n}\right) \rightarrow \Phi, \quad \text { when } n \rightarrow+\infty .
$$

Let $\left(V_{t}\right)_{t \in[0, T]}$ be a continuous adapted process valued in $\overline{\mathcal{D}}$. From the convexity property of $\mathcal{D}$ and the fact that $\nabla \varphi_{n}\left(Y^{n}\right)=n\left(Y^{n}-\mathfrak{P}\left(Y_{s}^{n}\right)\right)$, recall $(3.9)$, we have

$$
\int_{0}^{T}\left(Y_{t}^{n}-V_{t}\right)^{\dagger} \nabla \varphi_{n}\left(Y_{t}^{n}\right) \mathrm{d} t \leqslant 0
$$

By strong convergence of $\left(Y^{n}\right)_{n \geqslant 0}$ to $Y$, weak convergence of $\left(\nabla \varphi_{n}\left(Y^{n}\right)\right)_{n \geqslant 0}$ and the uniform $L^{2}$-bound on $\nabla \varphi_{n}\left(Y^{n}\right)$, recall Lemma 3.2, we obtain

$$
\mathbb{E}\left[\int_{0}^{T}\left(Y_{t}-V_{t}\right)^{\dagger} \Phi_{t} \mathrm{~d} t \mathbf{1}_{A}\right] \leqslant 0
$$

for all $A \in \mathcal{F}_{T}$. This leads to $\int_{0}^{T}\left(Y_{t}-V_{t}\right)^{\dagger} \Phi_{t} \mathrm{~d} t \leqslant 0$. Using Lemma 2.1 in [8] $\omega$-wise, we obtain that

$$
\Phi \in \partial \varphi(Y) \text { and } \int_{0}^{T} \mathbf{1}_{\left\{Y_{t} \notin \partial \mathcal{D}\right\}}\left|\Phi_{t}\right| \mathrm{d} t=0
$$


which fully characterise $\Phi$.

2.b Now we want to show that $(Y, Z, \Phi)$ is solution of $(3.8)$. By strong convergence of $\left(Y^{n}, Z^{n}\right)$ to $(Y, Z)$ and the Lipschitz-continuity of $f$, we have

$$
f\left(\cdot, Y^{n}, Z_{.}^{n}\right) \stackrel{\mathscr{H}^{2}}{\longrightarrow} f(\cdot, Y ., Z .) \text { and } \int_{0}^{t} Z_{s}^{n} \mathrm{~d} W_{s} \stackrel{\mathscr{L}^{2}}{\longrightarrow} \int_{0}^{t} Z_{s} \mathrm{~d} W_{s}
$$

for all $t \leqslant T$. Moreover, $\Phi^{n} \rightarrow \Phi$ in $L^{2}([0, T] \times \Omega)$, when $n \rightarrow+\infty$. Using Mazur's Lemma, we know that there exists a convex combination of the above converging strongly in $L^{2}([0, T] \times \Omega)$, namely

$$
{ }^{p} \Phi:=\sum_{r=p}^{N_{p}} \lambda_{r}^{p} \Phi^{r} \stackrel{p \rightarrow \infty}{\rightarrow} \Phi
$$

where $\lambda_{r}^{p} \geqslant 0$ for all $p \in \mathbb{N}$ and $p \leqslant r \leqslant N_{p}$, and $\sum_{r=p}^{N_{p}} \lambda_{r}^{p}=1$. Let us observe that by strong convergence, the following combination

$$
\left({ }^{p} Y,{ }^{p} Z\right):=\sum_{r=p}^{N_{p}} \lambda_{r}^{p}\left(Y^{r}, Z^{r}\right)
$$

still converges to $(Y, Z)$ in $\mathscr{S}^{2} \times \mathscr{H}^{2}$ and, by strong convergence,

$$
\sum_{r=p}^{N_{p}} \lambda_{r}^{p} f\left(\cdot, Y^{r}, Z^{r}\right) \stackrel{\mathscr{H}^{2}}{\longrightarrow} f(\cdot, Y, Z) \text { and } \int_{0}^{t}{ }^{p} Z_{s} \mathrm{~d} W_{s} \stackrel{\mathscr{L}^{2}}{\longrightarrow} \int_{0}^{t} Z_{s} \mathrm{~d} W_{s}, t \leqslant T .
$$

Moreover, we remark that

$$
\sum_{r=p}^{N_{p}} \lambda_{r}^{p} H\left(., Y^{r}\right) \Phi^{r}=\sum_{r=p}^{N_{p}} \lambda_{r}^{p}\left[H\left(., Y^{r}\right)-H(., Y)\right] \Phi^{r}+H(., Y)^{p} \Phi .
$$

Using the Lipschitz property of $H$ and the uniform $L^{2}$-bound on $\nabla \varphi_{n}\left(Y^{n}\right)$, the first term in the right hand side of the previous equation tends to zero in $\mathscr{H}^{2}$. Then we get

$$
\sum_{r=p}^{N_{p}} \lambda_{r}^{p} H\left(., Y^{r}\right) \Phi^{r} \stackrel{\mathscr{H}^{2}}{\longrightarrow} H(., Y) \Phi .
$$

Finally, we just have to pass to the limit into

$$
{ }^{p} Y_{t}=\xi+\int_{t}^{T} \sum_{r=p}^{N_{p}} \lambda_{r}^{p} f\left(s, Y_{s}^{r}, Z_{s}^{r}\right) \mathrm{d} s-\int_{t}^{T}{ }^{p} Z_{s} \mathrm{~d} W_{s}-\int_{t}^{T} \sum_{r=p}^{N_{p}} \lambda_{r}^{p} H\left(s, Y_{s}^{r}\right) \Phi_{s}^{r} \mathrm{~d} s
$$

to conclude the proof of the theorem. 


\subsection{General case}

Theorem 3.1. Assume that $(\boldsymbol{S B})$ holds and $\xi \in \mathfrak{T}_{\mathfrak{B}(\Lambda)}$. There exists a unique solution $(Y, Z, \Phi) \in \mathscr{S}^{2} \times \mathscr{B}^{2} \times \mathscr{B}^{2}$ to 1.1$)$.

Before proving our main result, we consider the following lemma which is a key result for the study of Obliquely Reflected BSDEs, as it proves, among other things, the structural condition 2.12). It is the counterpart of Lemma 3.2 introduced for the penalised BSDE.

Lemma 3.3. Assume that (SB) holds. Let $(Y, Z, \Phi) \in \mathscr{S}^{2} \times \mathscr{H}^{2} \times \mathscr{H}^{2}$ be a solution to the Obliquely Reflected BSDE (1.1). Then, the structural condition (2.12) holds true for $(Y, Z, \Phi)$ for some $K>0$. Moreover, there exists $c:=c\left(\sigma^{\xi}\right)$ such that

$$
\|\Phi\|_{\mathscr{B}^{2}} \leqslant c .
$$

Proof. Applying Itô's formula to $U_{t}:=\phi\left(Y_{t}\right)$, recall assumption (SB), we compute that $\mathrm{d} U_{t}=a_{t} \mathrm{~d} t+b_{t} \mathrm{~d} W_{t}$ with

$$
a_{t}:=\partial \phi\left(Y_{t}\right)\left\{-f\left(t, Y_{t}, Z_{t}\right)+H\left(t, Y_{t}\right) \Phi_{t}\right\}+\frac{1}{2} \operatorname{Tr}\left[\partial^{2} \phi\left(Y_{t}\right) Z_{t} Z_{t}^{*}\right] \quad \text { and } \quad b_{t}:=\partial \phi\left(Y_{t}\right) Z_{t} .
$$

Using Itô-Tanaka formula, we obtain

$$
\mathrm{d}\left[-U_{t}\right]^{+}=-a_{t} 1_{\left\{U_{t}<0\right\}} \mathrm{d} t-b_{t} 1_{\left\{U_{t}<0\right\}} \mathrm{d} W_{t}+\mathrm{d} L_{t}^{0}
$$

where $L^{0}$ is the local time at 0 of the semi-martingale $U$. Taking the difference of the two previous equations, we obtain

$$
0=a_{t} 1_{\left\{U_{t}=0\right\}} \mathrm{d} t+b_{t} 1_{\left\{U_{t}=0\right\}} \mathrm{d} W_{t}+\mathrm{d} L_{t}^{0}
$$

which leads to $a_{t} 1_{\left\{U_{t}=0\right\}} \mathrm{d} t \leqslant 0$. We then deduce

$$
\left|\Phi_{t}\right| \mathrm{d} t \leqslant \frac{1}{\eta}\left[\partial \phi\left(Y_{t}\right) f\left(t, Y_{t}, Z_{t}\right)\right]^{+} \mathrm{d} t
$$

recall $(2.9)$ and Remark $2.3 i)$. From this, we deduce that a fortiori $(2.12)$ holds true.

We should notice that in the proof of the above lemma, we obtain a stronger result than the structural condition (2.12). Indeed, we are able to control in (3.16) the reflecting process without the conditional expectation appearing in (2.12).

We now turn to the proof of our main result for this section.

\section{Proof of Theorem 3.1}

1. We first prove uniqueness of the solution. Let $\left({ }^{1} Y,{ }^{1} Z,{ }^{1} \Phi\right)$ and $\left({ }^{2} Y,{ }^{2} Z,{ }^{2} \Phi\right)$ be two solutions of (3.8) in $\mathscr{S}^{2} \times \mathscr{H}^{2} \times \mathscr{H}^{2}$. We first observe that both solutions satisfies (2.12) by application of Lemma 3.3 which allows us to invoke Proposition 2.1. Moreover, both solutions satisfy 2.28 by definition. Then, a straightforward application of Proposition 
2.2 concludes the proof of this step, noticing that all the terms in the right hand side of 2.29 are null.

2. We now turn to the existence question.

2.a We first approximate $\xi$ by a sequence of bounded random variables $\left(\xi_{N}\right)_{N \geqslant 1}$. Let $\left(\tau_{N}\right)_{N \geqslant 1}$ be the sequence of stopping time defined by

$$
\tau_{N}:=\inf \left\{t \geqslant 0|| \mathcal{Y}_{t}^{\xi} \mid \geqslant N\right\} \wedge T,
$$

and we set $\xi_{N}:=\mathcal{Y}_{\tau_{N}}^{\xi}$. Importantly, we observe that $\xi_{N}$ satisfies (SB)(i) and it belongs also to the class $\mathfrak{T}_{\mathfrak{B}(\Lambda)}$, indeed $\int_{0}^{T}\left|\mathcal{Z}_{s}^{\xi_{N}}\right|^{2} \mathrm{~d} s \leqslant \int_{0}^{T}\left|\mathcal{Z}_{s}^{\xi}\right|^{2} \mathrm{~d} s$. For later use, let us also remark that

$$
\sigma^{\xi^{N}} \leqslant \sigma^{\xi}, \text { for all } N \geqslant 1
$$

recall (2.8). Moreover, since

$$
\xi_{N} \rightarrow \xi \mathbb{P}-\text { a.s. } \quad \text { and } \quad\left|\xi_{N}-\xi\right| \leqslant 2 \sup _{t \in[0, T]}\left|\mathcal{Y}_{t}^{\xi}\right|,
$$

we have that by the dominated convergence theorem, recall Remark 2.2 (i), $\xi_{N} \rightarrow \xi$ in $\mathscr{L}^{q}$, for any $q \geqslant 1$.

2.b Applying Proposition 3.1, we introduce a sequence of Obliquely RBSDEs, $\left(Y^{N}, Z^{N}, \Phi^{N}\right)$ with terminal condition $\xi_{N}$. We now show that $\left(Y^{N}, Z^{N}\right)$ is a Cauchy sequence in $\mathscr{S}^{2} \times \mathscr{H}^{2}$. First, we apply the stability estimate given in Proposition 2.2 for $N, P \geqslant 1$, we have

$$
\sup _{t \in[0, T]} \mathbb{E}\left[\left|Y_{t}^{N}-Y_{t}^{P}\right|^{2}\right]+\left\|Z^{N}-Z^{P}\right\|_{\mathscr{H}^{2}}^{2} \leqslant C \mathbb{E}\left[\Gamma_{T}^{N, P}\left|\xi^{N}-\xi^{P}\right|^{2}\right],
$$

with $\Gamma^{N, P}$ such that for some $p>1$ and $C>0$,

$$
\mathbb{E}\left[\left|\Gamma_{T}^{N, P}\right|^{p}\right] \leqslant C,
$$

where importantly $p$ and $C$ do not depend on $(N, P)$, recall (3.17). Using Hölder inequality, we then obtain

$$
\sup _{t \in[0, T]} \mathbb{E}\left[\left|Y_{t}^{N}-Y_{t}^{P}\right|^{2}\right]+\left\|Z^{N}-Z^{P}\right\|_{\mathscr{H}^{2}}^{2} \leqslant C\left\|\xi^{N}-\xi^{P}\right\|_{\mathscr{L}^{2 q}}^{2} .
$$

Following classical arguments, see Step 1.a in the proof of Proposition 3.1, we compute also

$$
\left\|Y^{N}-Y^{P}\right\|_{\mathscr{S}^{2}}^{2} \leqslant C \mathbb{E}\left[\int_{0}^{T}\left|Y_{t}^{N}-Y_{t}^{P}\right|\left(\left|\Phi_{t}^{N}\right|+\left|\Phi_{t}^{P}\right|\right) \mathrm{d} t\right]+C\left\|Z^{N}-Z^{P}\right\|_{\mathscr{H}^{2}}^{2} .
$$

Applying Cauchy-Schwarz inequality, and combining Lemma 3.3 and (3.17), we get

$$
\left\|Y^{N}-Y^{P}\right\|_{\mathscr{S}^{2}}^{2} \leqslant C\left(\left\|Y^{N}-Y^{P}\right\|_{\mathscr{H}^{2}}+\left\|Z^{N}-Z^{P}\right\|_{\mathscr{H}^{2}}^{2}\right) .
$$


Eventually, we obtain

$$
\left\|Y^{N}-Y^{P}\right\|_{\mathscr{S}^{2}}^{2}+\left\|Z^{N}-Z^{P}\right\|_{\mathscr{H}^{2}}^{2} \leqslant C\left(\left\|\xi^{N}-\xi^{P}\right\|_{\mathscr{L}^{2 q}}+\left\|\xi^{N}-\xi^{P}\right\|_{\mathscr{L}^{2 q}}^{2}\right) .
$$

From the conclusion of Step 1. we deduce the Cauchy property of the sequence $\left(Y^{N}, Z^{N}\right)$ and we denote $(Y, Z)$ its limit. The proof is then concluded following the same arguments as in step 2 of Proposition 3.1. once observed that by Lemma 3.3 .

$$
\mathbb{E}\left[\int_{0}^{T}\left|\Phi_{s}^{N}\right|^{2} \mathrm{~d} s\right] \leqslant C,
$$

where again $C$ does not depend on $N$ from (3.17).

\section{A general existence result in the Markovian framework}

In this section, we introduce a Markovian framework: for all $(t, x) \in[0, T] \times \mathbb{R}^{q}$, we denote $\left(X_{s}^{t, x}\right)_{s \in[0, T]}$ the solution of the SDE

$$
\begin{aligned}
\mathrm{d} X_{s} & =b\left(s, X_{s}\right) \mathrm{d} s+\sigma\left(s, X_{s}\right) \mathrm{d} W_{s}, \quad s \in[t, T], \\
X_{s} & =x, \quad s \in[0, t],
\end{aligned}
$$

and we consider the following Markovian reflected BSDE:

$$
\left\{\begin{array}{l}
Y_{t}=g\left(X_{T}^{0, a}\right)+\int_{t}^{T} f\left(s, X_{s}^{0, a}, Y_{s}, Z_{s}\right) \mathrm{d} s-\int_{t}^{T} Z_{s} \mathrm{~d} W_{s}-\int_{t}^{T} H\left(s, X_{s}^{0, a}, Y_{s}, Z_{s}\right) \Phi_{s} d s \\
Y_{t} \in \overline{\mathcal{D}}, \quad \Phi_{t} \in \partial \varphi\left(Y_{t}\right), \quad 0 \leqslant t \leqslant T, \quad \int_{0}^{T} \mathbf{1}_{\left\{Y_{t} \notin \partial \mathcal{D}\right\}}\left|\Phi_{t}\right| \mathrm{d} t=0 .
\end{array}\right.
$$

The main goal of this section is to prove an existence result for the above reflected BSDE when $H$ is only continuous, compare with assumption (SB). We also discuss the case of discontinuous $H$ and the difficulty arising for uniqueness in this setting.

\subsection{Continuous oblique direction of reflection}

We now introduce the main setting for this part. The set of assumption below echoes assumption (A) introduced in Section 2.1 but in a Markovian setting.

\section{Assumption (AM)}

i) $b:[0, T] \times \mathbb{R}^{q} \rightarrow \mathbb{R}^{q}$ and $\sigma:[0, T] \times \mathbb{R}^{q} \rightarrow \mathbb{R}^{q \times k}$ are measurable functions satisfying linear growth condition and uniform Lipschitz condition with respect to the space variable namely

$|b(t, x)|+|\sigma(t, x)| \leqslant L(1+|x|)$ and $|b(t, x)-b(t, y)|+|\sigma(t, x)-\sigma(t, y)| \leqslant L|x-y|$, for some $L>0$ and all $(t, x, y) \in[0, T] \times \mathbb{R}^{q} \times \mathbb{R}^{q}$. 
ii) $g: \mathbb{R}^{q} \rightarrow \mathbb{R}^{d}$ is a measurable function and there exists $p \in \mathbb{R}^{+}$such that for any $x \in \mathbb{R}^{q}$,

$$
|g(x)| \leqslant L\left(1+|x|^{p}\right)
$$

iii) $f:[0, T] \times \mathbb{R}^{q} \times \mathbb{R}^{d} \times \mathbb{R}^{d \times k} \rightarrow \mathbb{R}^{d}$ is a measurable function satisfying: there exists $p \in \mathbb{R}^{+}$such that, for any $(t, x, y, z) \in[0, T] \times \mathbb{R}^{q} \times \mathbb{R}^{d} \times \mathbb{R}^{d \times k}$, we have

$$
|f(t, x, y, z)| \leqslant L\left(1+|x|^{p}+|y|+|z|\right),
$$

and, for all $(t, x) \in[0, T] \times \mathbb{R}^{d}, f(t, x, .,$.$) is continuous on \mathbb{R}^{d} \times \mathbb{R}^{d \times k}$.

iv) $H:[0, T] \times \mathbb{R}^{q} \times \mathbb{R}^{d} \times \mathbb{R}^{d \times k} \rightarrow \mathbb{R}^{d \times d}$ is a measurable function. There exists $\eta>0$ such that, for all $(t, x, y, z) \in[0, T] \times \mathbb{R}^{q} \times \mathbb{R}^{d} \times \mathbb{R}^{d \times k}$

$$
\begin{gathered}
H(t, x, \mathfrak{P}(y), z) v \cdot v \geqslant \eta, \quad \forall v \in \mathfrak{n}(\mathfrak{P}(y)), \\
\text { and }|H(t, x, \mathfrak{P}(y), z)| \leqslant L .
\end{gathered}
$$

v) Let $\mathcal{X}=\left\{\mu(t, x ; s, d y), x \in \mathbb{R}^{q}\right.$ and $\left.0 \leqslant t \leqslant s \leqslant T\right\}$ be the family of laws of $X^{t, x}$ on $\mathbb{R}^{q}$, i.e., the measures such that $\forall A \in \mathcal{B}\left(\mathbb{R}^{q}\right), \mu(t, x ; s, A)=\mathbb{P}\left(X_{s}^{t, x} \in A\right)$. For any $t \in[0, T)$, for any $\mu(0, a ; t, d y)$-almost every $x \in \mathbb{R}^{q}$, and any $\left.\left.\delta \in\right] 0, T-t\right]$, there exists an application $\phi_{t, x}:[t, T] \times \mathbb{R}^{d} \rightarrow \mathbb{R}^{+}$such that:

(a) $\forall k \geqslant 1, \phi_{t, x} \in L^{2}\left([t+\delta, T] \times[-k, k]^{q} ; \mu(0, a ; s, d y) d s\right)$,

(b) $\mu(t, x ; s, d y) d s=\phi_{t, x}(s, y) \mu(0, a ; s, d y) d s$ on $[t+\delta, T] \times \mathbb{R}^{q}$.

vi) For all $(t, x) \in[0, T] \times \mathbb{R}^{d}, H(t, x, . .$.$) is continuous on \mathbb{R}^{d} \times \overline{\mathcal{D}}$.

Remark 4.1. i) We observe that $H(t, X, \cdot)$ and $f(t, X, \cdot)$ satisfy assumption (A). Thus we will use in the sequel the a priori estimates obtained in Section 2.2.

ii) Remark 2.1 applies for $H$ which is continuous in this context.

iii) The $\mathscr{L}^{2}$-domination condition $(\mathbf{A M})(v)$ was initially introduced in [10]. We refer to [10, 4] for examples of assumptions on coefficients of the SDE (4.1] under which $(\mathbf{A M})(v)$ is true.

Theorem 4.1. Assume (AM). Then, there exists a solution $(Y, Z) \in \mathscr{S}^{2} \times \mathscr{H}^{2}$ to (4.2). Moreover, the following Markovian representation holds true:

There exist $u:[0, T] \times \mathbb{R}^{q} \rightarrow \mathbb{R}^{d}$ and $v:[0, T] \times \mathbb{R}^{q} \rightarrow \mathbb{R}^{d \times k}$ measurable functions such that

$$
Y_{t}=u\left(t, X_{t}^{0, a}\right) \quad \text { and } \quad Z_{t}=v\left(t, X_{t}^{0, a}\right),
$$

and, for some $c>0$, for all $(t, x) \in[0, T] \times \mathbb{R}^{q}$,

$$
|u(t, x)| \leqslant c\left(1+|x|^{p}\right) .
$$

By choosing properly the function $H$ we can obtain the following corollary. 
Corollary 4.1. Let us consider the following obliquely reflected Markovian BSDE

$$
\begin{cases}Y_{t}=g\left(X_{T}^{0, a}\right)+\int_{t}^{T} f\left(s, X_{s}^{0, a}, Y_{s}, Z_{s}\right) \mathrm{d} s-\int_{t}^{T} Z_{s} \mathrm{~d} W_{s}+\int_{t}^{T} \Psi_{s} \mathrm{~d} s, & 0 \leqslant t \leqslant T, \\ Y_{t}^{\ell} \geqslant \max _{j \in \mathcal{I}}\left\{Y_{t}^{j}-c^{\ell j}\right\}, & 0 \leqslant t \leqslant T, \ell \in \mathcal{I}, \\ \int_{0}^{T}\left[Y_{t}^{\ell}-\max _{j \in \mathcal{I} \backslash\{\ell\}}\left\{Y_{t}^{j}-c^{\ell j}\right\}\right] \Psi_{t}^{\ell} \mathrm{d} t=0, & \ell \in \mathcal{I},\end{cases}
$$

where $\mathcal{I}:=\{1, \ldots, d\}$ and the switching costs $\left(c^{i j}\right)_{i, j \in \mathcal{I}}$ satisfy the following structure condition

$$
\begin{cases}c^{i i}=0, & \text { for } 1 \leqslant i \leqslant d \\ \left\{c^{i j}+c^{j l}-c^{i l}\right\}>0, & \text { for } 1 \leqslant i, j \leqslant d \text { with } i \neq j, j \neq l .\end{cases}
$$

We assume that assumption $(\boldsymbol{A} \boldsymbol{M})$ is in force. Then there exists a solution $(Y, Z, \Phi) \in$ $\mathscr{S}^{2} \times \mathscr{H}^{2} \times \mathscr{H}^{2}$ to (4.4). Moreover the following Markovian representation holds true: There exist two measurable functions $u:[0, T] \times \mathbb{R}^{q} \rightarrow \mathbb{R}^{d}$ and $v:[0, T] \times \mathbb{R}^{q} \rightarrow \mathbb{R}^{d \times k}$ such that

$$
Y_{t}=u\left(t, X_{t}^{0, x}\right) \quad \text { and } \quad Z_{t}=v\left(t, X_{t}^{0, x}\right),
$$

and, for some $c>0$, for all $(t, x) \in[0, T] \times \mathbb{R}^{q}$,

$$
|u(t, x)| \leqslant c\left(1+|x|^{p}\right) .
$$

Remark 4.2. The main novelty here is the dependence of the generator on the whole $z$ (as in the concomitant article [4]) which extend the result of [13, 12, 1] and the possibility to consider negative switching costs. We refer to [17] and references inside for a recent work dealing with switching problems with signed switching costs. Our result only cover the case of constant switching costs due to a priori estimates obtained previously in the framework of a deterministic domain $\mathcal{D}$. Nevertheless our approach might be adapted to treat random domains and then tackle the problem of switched BSDEs with random signed switching costs.

Before giving the proof of Theorem 4.1 and Corollary 4.1 , we start by considering an approximation of 4.2 . Let $\theta$ be an element of $C^{\infty}\left(\mathbb{R}^{d+d \times k}, \mathbb{R}^{+}\right)$with compact support and satisfying

$$
\int_{\mathbb{R}^{d+d \times k}} \theta(y, z) \mathrm{d} y \mathrm{~d} z=1 .
$$

For all $n \in \mathbb{N}$ and $(t, x, y, z) \in[0, T] \times \mathbb{R}^{q} \times \mathbb{R}^{d} \times \mathbb{R}^{d \times k}$ we set

$$
\begin{aligned}
f_{n}(t, x, y, z) & =\int_{\mathbb{R}^{d+d \times k}} n^{2} f(t, x, y, z) \theta(n(y-u), n(z-v)) \mathrm{d} u \mathrm{~d} v \\
H_{n}(t, x, y, z) & =\int_{\mathbb{R}^{d+d \times k}} n^{2} H(t, x, y, z) \theta(n(y-u), n(z-v)) \mathrm{d} u \mathrm{~d} v .
\end{aligned}
$$

By classical convolution arguments functions $\left(f_{n}\right)_{n \in \mathbb{N}}$ and $\left(H_{n}\right)_{n \in \mathbb{N}}$ satisfy following properties. 
Lemma 4.1. Assume (AM).

i) $f_{n}:[0, T] \times \mathbb{R}^{q} \times \mathbb{R}^{d} \times \mathbb{R}^{d \times k} \rightarrow \mathbb{R}^{d}$ and $H_{n}:[0, T] \times \mathbb{R}^{q} \times \mathbb{R}^{d} \times \mathbb{R}^{d \times k} \rightarrow \mathbb{R}^{d \times d}$ are measurable and uniformly Lipschitz functions with respect to $(y, z)$.

ii) $\left|f_{n}(t, x, y, z)\right| \leqslant L\left(1+|x|^{p}+|y|+|z|\right)$ and $\left|H_{n}(t, x, y, z)\right| \leqslant L$ for all $(t, x, y, z) \in$ $[0, T] \times \mathbb{R}^{q} \times \mathbb{R}^{d} \times \mathbb{R}^{d \times k}$.

iii) For all $(t, x) \in[0, T] \times \mathbb{R}^{q}$ and $\mathcal{K}$ a compact subset of $\mathbb{R}^{d} \times \mathbb{R}^{d \times k}$

$$
\sup _{(y, z) \in \mathcal{K}}\left|f_{n}(t, x, y, z)-f(t, x, y, z)\right|+\sup _{(y, z) \in \mathcal{K}}\left|H_{n}(t, x, y, z)-H(t, x, y, z)\right| \stackrel{n \rightarrow+\infty}{\longrightarrow} 0 .
$$

For any $n \in \mathbb{N}$, we then consider the following BSDE

$$
\begin{aligned}
Y_{t}^{n}= & g\left(X_{T}^{0, a}\right)+\int_{t}^{T} f_{n}\left(s, X_{s}^{0, a}, Y_{s}^{n}, Z_{s}^{n}\right) \mathrm{d} s \\
& -\int_{t}^{T} Z_{s}^{n} \mathrm{~d} W_{s}-\int_{t}^{T} H_{n}\left(s, X_{s}^{0, a}, Y_{s}^{n}, Z_{s}^{n}\right) \nabla \varphi_{n}\left(Y_{s}^{n}\right) \mathrm{d} s, \quad t \in[0, T]
\end{aligned}
$$

where $\varphi_{n}$ is defined in 1.3 with $M$ fixed to an arbitrary value. Note that, in this section, for the reader's convenience, we write simply $\varphi_{n}$ instead of $\varphi_{n}^{M}$.

Lemma 4.2. There exists a unique solution to 4.6 in $\mathscr{S}^{2} \times \mathscr{H}^{2}$. Moreover, there is a Markovian representation for this solution: for all $n \in \mathbb{N}$, there exist $u_{n}:[0, T] \times \mathbb{R}^{q} \rightarrow$ $\mathbb{R}^{d}$ and $v_{n}:[0, T] \times \mathbb{R}^{q} \rightarrow \mathbb{R}^{d \times k}$ measurable functions satisfying

$$
Y_{t}^{n}=u_{n}\left(t, X_{T}^{0, a}\right) \text { and } Z_{t}^{n}=v_{n}\left(t, X_{T}^{0, a}\right) .
$$

Moreover, for all $(t, x) \in[0, T] \times \mathbb{R}^{q},\left(u_{n}\left(s, X_{s}^{t, x}\right), v_{n}\left(s, X_{s}^{t, x}\right)\right)_{s \in[t, T]}$ is the unique solution in $\mathscr{S}^{2} \times \mathscr{H}^{2}$ of the BSDE

$$
\begin{aligned}
Y_{s}^{n, t, x}= & g\left(X_{T}^{t, x}\right)+\int_{s}^{T} f_{n}\left(r, X_{r}^{t, x}, Y_{r}^{n, t, x}, Z_{r}^{n, t, x}\right) \mathrm{d} r-\int_{s}^{T} Z_{r}^{n, t, x} \mathrm{~d} W_{r} \\
& -\int_{s}^{T} H_{n}\left(r, X_{r}^{t, x}, Y_{r}^{n, t, x}, Z_{r}^{n, t, x}\right) \nabla \varphi_{n}\left(Y_{r}^{n, t, x}\right) \mathrm{d} r \quad s \in[t, T] .
\end{aligned}
$$

Proof. We use the same arguments as in the proof of Lemma 3.1 Since $H_{n}$ and $\nabla \varphi_{n}$ are two Lipschitz bounded functions (with respect to $y$ and $z$ ), the penalised BSDE (4.6) has a Lipschitz driver and the classical theory then applies to get the existence, uniqueness and representation result.

By applying Lemma 2.1 and Lemma 3.2, we obtain the following estimates for $\left(Y^{n, t, x}, Z^{n, t, x}\right)$.

Proposition 4.1. For all $(t, x) \in[0, T] \times \mathbb{R}^{q}$, we have

$$
\sup _{t \leqslant s \leqslant T} \mathbb{E}\left[\left|Y_{s}^{n, t, x}\right|^{2}+\varphi_{n}\left(Y_{s}^{n, t, x}\right)\right]+\mathbb{E}\left[\int_{t}^{T}\left|Z_{s}^{n, t, x}\right|^{2} \mathrm{~d} s+\int_{t}^{T}\left|\nabla \varphi_{n}\left(Y_{s}^{n, t, x}\right)\right|^{2} \mathrm{~d} s\right] \leqslant C\left(1+|x|^{2 p}\right) .
$$


In particular, Proposition 4.1 yields that, for some $c>0$,

$$
\left|u_{n}(t, x)\right| \leqslant c\left(1+|x|^{p}\right), \quad \forall n \in \mathbb{N}, \quad \forall(t, x) \in[0, T] \times \mathbb{R}^{q} .
$$

We now turn to the proof of the main result for this section.

\section{Proof of Theorem 4.1}

The proof follows mainly from arguments in [10]. Some extra work is required to identify the reflecting process properly.

1. Define,

$$
F_{n}(t, x)=f_{n}\left(t, x, u_{n}(t, x), v_{n}(t, x)\right), \quad G_{n}(t, x)=H_{n}\left(t, x, u_{n}(t, x), v_{n}(t, x)\right) \nabla \varphi_{n}\left(u_{n}(t, x)\right),
$$

and

$$
\mathfrak{F}_{n}:=F_{n}-G_{n},
$$

we compute

$$
\begin{aligned}
\int_{\mathbb{R}^{q}} \int_{0}^{T}\left|\mathfrak{F}_{n}(s, y)\right|^{2} \mu(0, a ; s, \mathrm{~d} y) \mathrm{d} s & =\mathbb{E}\left[\int_{0}^{T}\left|\mathfrak{F}_{n}\left(s, X_{s}^{0, a}\right)\right|^{2} \mathrm{~d} s\right] \\
& \leqslant \mathbb{E}\left[\int_{0}^{T} C\left(1+\left|X_{s}^{0, a}\right|^{2 p}+\left|Y_{s}^{n}\right|^{2}+\left|Z_{s}^{n}\right|^{2}+\left|\nabla \varphi_{n}\left(Y_{s}^{n}\right)\right|^{2}\right) \mathrm{d} s\right] \\
& \leqslant C,
\end{aligned}
$$

by using Proposition 4.1 . Thus we get $\mathfrak{F}_{n} \rightarrow \mathfrak{F}$ in $L^{2}\left([0, T] \times \mathbb{R}^{q} ; \mu(0, a ; s, \mathrm{~d} x) \mathrm{d} s\right)$, up to a subsequence.

2. We now show that $\left(u_{n}(t, x)\right)_{n \in \mathbb{N}}$ is a Cauchy sequence in $\mathbb{R}^{d}$ for all $t \in[0, T]$ and for $\mu(0, a ; t, \mathrm{~d} x)$-almost every $x \in \mathbb{R}^{q}$. When $t=T$ the sequence is constant and the result is obvious. When $t<T, x \in \mathbb{R}^{q}$ and $\delta \in(0, T-t]$, we compute

$$
\begin{aligned}
\left|u_{n}(t, x)-u_{m}(t, x)\right| & =\left|\mathbb{E}\left[\int_{t}^{T}\left(\mathfrak{F}_{n}\left(s, X_{s}^{t, x}\right)-\mathfrak{F}_{m}\left(s, X_{s}^{t, x}\right)\right) \mathrm{d} s\right]\right| \\
& \leqslant \mathbb{E}\left[\int_{t}^{t+\delta}\left|\mathfrak{F}_{n}\left(s, X_{s}^{t, x}\right)-\mathfrak{F}_{m}\left(s, X_{s}^{t, x}\right)\right| \mathrm{d} s\right]=: A_{1} \\
& +\mathbb{E}\left[\int_{t+\delta}^{T}\left|\mathfrak{F}_{n}\left(s, X_{s}^{t, x}\right)-\mathfrak{F}_{m}\left(s, X_{s}^{t, x}\right)\right| \mathbf{1}_{\left\{\left|X_{s}^{t, x}\right| \geqslant \kappa\right\}} \mathrm{d} s\right]=: A_{2} \\
& +\left|\mathbb{E}\left[\int_{t+\delta}^{T}\left(\mathfrak{F}_{n}\left(s, X_{s}^{t, x}\right)-\mathfrak{F}_{m}\left(s, X_{s}^{t, x}\right)\right) \mathbf{1}_{\left\{\left|X_{s}^{t, x}\right|<\kappa\right\}} \mathrm{d} s\right]\right|=: A_{3} .
\end{aligned}
$$

For the first two terms, we easily get

$$
\begin{aligned}
& A_{1} \leqslant \delta^{\frac{1}{2}} \mathbb{E}\left[\int_{t}^{t+\delta}\left|\mathfrak{F}_{n}\left(s, X_{s}^{t, x}\right)-\mathfrak{F}_{m}\left(s, X_{s}^{t, x}\right)\right|^{2} \mathrm{~d} s\right]^{\frac{1}{2}} \leqslant C\left(1+|x|^{p}\right) \delta^{\frac{1}{2}} \\
& A_{2} \leqslant C \kappa^{-\frac{1}{2} \mathbb{E}}\left[\int_{t+\delta}^{T}\left|X_{s}^{t, x}\right| \mathrm{d} s\right] \mathbb{E}\left[\int_{t+\delta}^{T}\left|\mathfrak{F}_{n}\left(s, X_{s}^{t, x}\right)-\mathfrak{F}_{m}\left(s, X_{s}^{t, x}\right)\right|^{2} \mathrm{~d} s\right]^{\frac{1}{2}} \leqslant C\left(1+|x|^{p+1}\right) \kappa^{-\frac{1}{2}},
\end{aligned}
$$


where $C$ is a constant that does not depend on $n$ nor $m$. For the third term, we have

$$
\begin{aligned}
A_{3} & =\left|\int_{\mathbb{R}^{q}} \int_{t+\delta}^{T}\left(\mathfrak{F}_{n}(s, y)-\mathfrak{F}_{m}(s, y)\right) \mathbf{1}_{\{|y| \leqslant \kappa\}} \mu(t, x ; s, \mathrm{~d} y) \mathrm{d} s\right| \\
& =\left|\int_{\mathbb{R}^{q}} \int_{t+\delta}^{T}\left(\mathfrak{F}_{n}(s, y)-\mathfrak{F}_{m}(s, y)\right) \mathbf{1}_{\{|y| \leqslant \kappa\}} \phi_{t, x}(s, y) \mu(0, a ; s, \mathrm{~d} y) \mathrm{d} s\right|
\end{aligned}
$$

for $\mu(0, a ; s, \mathrm{~d} x)$-almost every $x \in \mathbb{R}^{q}$, where we used the $L^{2}$-domination assumption. By weak convergence, $A_{3} \rightarrow 0$ when $n, m \rightarrow \infty$. Thus, by taking $\delta \rightarrow 0$ and $\kappa \rightarrow+\infty$ we show that for all $t \in[0, T]$ and for $\mu(0, a ; t, \mathrm{~d} x)$-almost every $x \in \mathbb{R}^{q},\left(u_{n}(t, x)\right)_{n \in \mathbb{N}}$ is a Cauchy sequence. So, there exists a Borelian application $u:[0, T] \times \mathbb{R}^{q} \rightarrow \mathbb{R}^{d}$ such that for all $t \in[0, T]$, for $\mu(0, a ; t, \mathrm{~d} x)$-almost every $x \in \mathbb{R}^{q}$,

$$
u(t, x)=\lim _{n \infty} u_{n}(t, x)
$$

We straightforwardly get, for all $t \in[0, T]$,

$$
Y_{t}^{n}=u_{n}\left(t, X_{t}^{0, a}\right) \rightarrow u\left(t, X_{t}^{0, a}\right):=Y_{t}, \quad \text { a.s. }
$$

and, observing that $\left|Y_{t}^{n}\right| \leqslant C\left(1+\left|X_{t}^{0, a}\right|^{p}\right)$, we obtain via the dominated convergence theorem, $Y_{t}^{n} \rightarrow Y_{t}$ in $L^{2}([0, T] \times \Omega, d t \otimes d \mathbb{P})$.

3. We can easily prove that the process $Y$ lives in the convex set $\overline{\mathcal{D}}$. Indeed, we have, recalling (3.1),

$$
\begin{aligned}
\sup _{0 \leqslant s \leqslant T} \mathbb{E}\left[\varphi_{1}\left(Y_{s}\right)\right] & \leqslant \sup _{0 \leqslant s \leqslant T} \mathbb{E}\left[\left|\varphi_{1}\left(Y_{s}\right)-\varphi_{1}\left(Y_{s}^{n}\right)\right|\right]+\frac{1}{n} \sup _{0 \leqslant s \leqslant T} \mathbb{E}\left[\varphi_{n}\left(Y_{s}^{n}\right)\right] \\
& \leqslant M \sup _{0 \leqslant s \leqslant T} \mathbb{E}\left[\left|Y_{s}-Y_{s}^{n}\right|\right]+\frac{C}{n} \stackrel{n \rightarrow+\infty}{\longrightarrow} 0
\end{aligned}
$$

where we used Proposition 4.1, the fact that $\varphi_{1}$ is a $M$-Lipschitz function and the convergence of $\left(Y^{n}\right)_{n \in \mathbb{N}}$. Then, for all $s \in[0, T], d\left(Y_{s}, \mathcal{D}\right)=0$ a.s. and so $Y_{s} \in \overline{\mathcal{D}}$ a.s.

4. We now show that $\left(Z^{n}\right)_{n}=\left(\left(v_{n}\left(t, X_{t}^{0, a}\right)\right)_{t \in[0, T]}\right)_{n}$ is a Cauchy sequence in $L^{2}([0, T] \times$ $\Omega, d t \otimes d \mathbb{P})$. For $n, m \geqslant 1$, we compute, applying Itô's formula,

$$
\begin{aligned}
\mathbb{E}\left[\int_{0}^{T}\left|Z_{s}^{n}-Z_{s}^{m}\right|^{2} \mathrm{~d} s\right] & \leqslant 2 \mathbb{E}\left[\int_{0}^{T}\left(Y_{t}^{n}-Y_{t}^{m}\right)\left(\mathfrak{F}_{n}\left(t, X_{t}^{0, a}\right)-\mathfrak{F}_{m}\left(t, X_{t}^{0, a}\right)\right) \mathrm{d} t\right] \\
& \leqslant C \mathbb{E}\left[\int_{0}^{T}\left|Y_{t}^{n}-Y_{t}^{m}\right|^{2} \mathrm{~d} t\right]^{\frac{1}{2}},
\end{aligned}
$$

which goes to 0 as $n, m \rightarrow \infty$. We denote by $Z$ the limit. From now on, we work with the progressively measurable version of $(Y, Z)$. 
5.a In the last step we have to prove that $(Y, Z)$ is a solution to BSDE (4.2). We start by studying the convergence of the generator. Firstly, we compute, for all $\kappa>0$,

$$
\begin{aligned}
& \mathbb{E}\left[\int_{0}^{T}\left|f_{n}\left(s, X_{s}^{0, a}, Y_{s}^{n}, Z_{s}^{n}\right)-f\left(s, X_{s}^{0, a}, Y_{s}, Z_{s}\right)\right| \mathrm{d} s\right] \\
\leqslant & \mathbb{E}\left[\int_{0}^{T}\left|f_{n}\left(s, X_{s}^{0, a}, Y_{s}^{n}, Z_{s}^{n}\right)-f\left(s, X_{s}^{0, a}, Y_{s}^{n}, Z_{s}^{n}\right)\right| \mathbf{1}_{\left\{\left|Y_{s}^{n}\right|+\left|Z_{s}^{n}\right| \leqslant \kappa\right\}} \mathrm{d} s\right]=: B_{1} \\
& +\mathbb{E}\left[\int_{0}^{T}\left|f_{n}\left(s, X_{s}^{0, a}, Y_{s}^{n}, Z_{s}^{n}\right)-f\left(s, X_{s}^{0, a}, Y_{s}^{n}, Z_{s}^{n}\right)\right| \mathbf{1}_{\left\{\left|Y_{s}^{n}\right|+\left|Z_{s}^{n}\right|>\kappa\right\}} \mathrm{d} s\right]=: B_{2} \\
& +\mathbb{E}\left[\int_{0}^{T}\left|f\left(s, X_{s}^{0, a}, Y_{s}^{n}, Z_{s}^{n}\right)-f\left(s, X_{s}^{0, a}, Y_{s}, Z_{s}\right)\right| \mathrm{d} s\right]=: B_{3} .
\end{aligned}
$$

Since $f$ and $f_{n}$ have a linear growth that does not depend on $n$, and $\left(Y^{n}, Z^{n}\right)$ is uniformly bounded in $L^{2}([0, T] \times \Omega, d t \otimes d P)$, we get, by using Markov inequality,

$$
B_{2} \leqslant \frac{C}{\kappa} \text {. }
$$

Moreover, we also get

$$
B_{3} \leqslant \mathbb{E}\left[\int_{0}^{T} \frac{\left|f\left(s, X_{s}^{0, a}, Y_{s}^{n}, Z_{s}^{n}\right)-f\left(s, X_{s}^{0, a}, Y_{s}, Z_{s}\right)\right|^{2}}{\left(1+\left|Y_{s}^{n}\right|+\left|Z_{s}^{n}\right|\right)^{2}} \mathrm{~d} s\right]^{1 / 2} \mathbb{E}\left[\int_{0}^{T}\left(1+\left|Y_{s}^{n}\right|+\left|Z_{s}^{n}\right|\right)^{2} \mathrm{~d} s\right]^{1 / 2}
$$

and thus, the dominated convergence theorem yields that $B_{3}$ converges to 0 as $n \rightarrow+\infty$. We now study the first term $B_{1}$. We have, for all $\kappa>0, s \in[0, T]$,

$$
\left|f_{n}\left(s, X_{s}^{0, a}, Y_{s}^{n}, Z_{s}^{n}\right)-f\left(s, X_{s}^{0, a}, Y_{s}^{n}, Z_{s}^{n}\right)\right| \mathbf{1}_{\left\{\left|Y_{s}^{n}\right|+\left|Z_{s}^{n}\right| \leqslant \kappa\right\}} \leqslant C\left(1+2 \kappa+\left|X_{s}^{0, a}\right|\right),
$$

and

$$
\begin{aligned}
& \left|f_{n}\left(s, X_{s}^{0, a}, Y_{s}^{n}, Z_{s}^{n}\right)-f\left(s, X_{s}^{0, a}, Y_{s}^{n}, Z_{s}^{n}\right)\right| \mathbf{1}_{\left\{\left|Y_{s}^{n}\right|+\left|Z_{s}^{n}\right| \leqslant \kappa\right\}} \\
\leqslant & \sup _{(y, z),|y|+|z| \leqslant \kappa}\left|f_{n}\left(s, X_{s}^{0, a}, y, z\right)-f\left(s, X_{s}^{0, a}, y, z\right)\right| .
\end{aligned}
$$

Thanks to Lemma 4.1(iii) we can assert that the second term of the last inequality converges to 0 and then, by applying the dominated convergence theorem, $B_{1}$ converges also to 0 . By taking $\kappa \rightarrow+\infty$, it follows that $\left(f_{n}\left(t, X_{t}^{0, a}, Y_{t}^{n}, Z_{t}^{n}\right)\right)_{t \in[0, T]}$ converges to $\left(f\left(t, X_{t}^{0, a}, Y_{t}, Z_{t}\right)\right)_{t \in[0, T]}$ in $L^{1}([0, T] \times \Omega, d t \otimes d P)$.

5.b Finally we study the reflecting term. Since

$$
\mathbb{E}\left[\int_{0}^{T}\left|\nabla \varphi_{n}\left(Y_{s}^{n}\right)\right|^{2} \mathrm{~d} s\right] \leqslant C,
$$

we have, up to a subsequence, the following weak $L^{2}([0, T] \times \Omega)$-convergence:

$$
\nabla \varphi_{n}\left(Y_{.}^{n}\right) \rightarrow \Phi, \quad \text { when } n \rightarrow+\infty,
$$


and we can follow step 2.a in the proof of Proposition 3.1 to obtain

$$
\Phi \in \partial \varphi(Y) \text { and } \int_{0}^{T} \mathbf{1}_{\left\{Y_{t} \notin \partial \mathcal{D}\right\}}\left|\Phi_{t}\right| \mathrm{d} t=0,
$$

which fully characterize $\Phi$. We now follow step 2.b in the proof of Proposition 3.1 Using Mazur's Lemma, we know that there exists a convex combination of $\left(\Phi^{n}\right)_{n \in \mathbb{N}}:=$ $\left(\nabla \varphi_{n}\left(Y^{n}\right)\right)_{n \in \mathbb{N}}$ converging strongly in $L^{2}([0, T] \times \Omega)$, namely

$$
{ }^{p} \Phi:=\sum_{r=p}^{N_{p}} \lambda_{r}^{p} \Phi^{r} \stackrel{p \rightarrow \infty}{\rightarrow} \Phi
$$

where $\lambda_{r}^{p} \geqslant 0$ for all $p \in \mathbb{N}$ and $p \leqslant r \leqslant N_{p}$, and $\sum_{r=p}^{N_{p}} \lambda_{r}^{p}=1$. Let us observe that by strong convergence, the following combination

$$
\left({ }^{p} Y,{ }^{p} Z\right):=\sum_{r=p}^{N_{p}} \lambda_{r}^{p}\left(Y^{r}, Z^{r}\right)
$$

still converges to $(Y, Z)$ in $\mathscr{S}^{2} \times \mathscr{H}^{2}$ and, by strong convergence again,

$$
\sum_{r=p}^{N_{p}} \lambda_{r}^{p} f_{r}\left(\cdot, X^{0, a}, Y^{r}, Z^{r}\right) \stackrel{L^{1}([0, T] \times \Omega, d t \otimes d P)}{\longrightarrow} f\left(\cdot, X^{0, a}, Y, Z\right) \text { and } \int_{0}^{t}{ }^{p} Z_{s} \mathrm{~d} W_{s} \stackrel{\mathscr{L}^{2}}{\longrightarrow} \int_{0}^{t} Z_{s} \mathrm{~d} W_{s} .
$$

Moreover, we remark that, for all $t \leqslant T$,

$$
\begin{aligned}
\mathcal{E}^{p}:= & \int_{0}^{t} \sum_{r=p}^{N_{p}} \lambda_{r}^{p} H_{r}\left(s, X_{s}^{0, a}, Y_{s}^{r}, Z_{s}^{r}\right) \Phi^{r} \mathrm{~d} s-\int_{0}^{t} H\left(s, X_{s}^{0, a}, Y_{s}, Z_{s}\right) \Phi_{s} \mathrm{~d} s \\
= & \int_{0}^{t} \sum_{r=p}^{N_{p}} \lambda_{r}^{p}\left\{H_{r}\left(s, X_{s}^{0, a}, Y_{s}^{r}, Z_{s}^{r}\right)-H\left(s, X_{s}^{0, a}, Y_{s}^{r}, Z_{s}^{r}\right)\right\} \Phi_{s}^{r} \mathrm{~d} s=: A_{1}^{p} \\
& +\int_{0}^{t} \sum_{r=p}^{N_{p}} \lambda_{r}^{p}\left\{H\left(s, X_{s}^{0, a}, Y_{s}^{r}, Z_{s}^{r}\right)-H\left(s, X_{s}^{0, a}, Y_{s}, Z_{s}\right)\right\} \Phi_{s}^{r} \mathrm{~d} s=: A_{2}^{p} \\
& +\int_{0}^{t} H\left(s, X_{s}^{0, a}, Y_{s}, Z_{s}\right)\left\{{ }^{p} \Phi_{s}-\Phi_{s}\right\} \mathrm{d} s=: A_{3}^{p} .
\end{aligned}
$$

We study each term in the right hand side of the above equality separately. For the first one, we compute using Cauchy-Schwartz inequality and the uniform bound on $\left\|\Phi^{n}\right\|_{\mathscr{H}^{2}}$

$$
\mathbb{E}\left[\left|A_{1}^{p}\right|\right] \leqslant C \sum_{r=p}^{N_{p}} \lambda_{r}^{p} \mathbb{E}\left[\int_{0}^{t}\left|H_{r}\left(s, X_{s}^{0, a}, Y_{s}^{r}, Z_{s}^{r}\right)-H\left(s, X_{s}^{0, a}, Y_{s}^{r}, Z_{s}^{r}\right)\right|^{2} \mathrm{~d} s\right]^{\frac{1}{2}} .
$$


For all $\kappa>0$, we then get

$$
\begin{aligned}
\mathbb{E}\left[\left|A_{1}^{p}\right|\right] & \leqslant C \sum_{r=p}^{N_{p}} \lambda_{r}^{p} \mathbb{E}\left[\int_{0}^{t}\left|H_{r}\left(s, X_{s}^{0, a}, Y_{s}^{r}, Z_{s}^{r}\right)-H\left(s, X_{s}^{0, a}, Y_{s}^{r}, Z_{s}^{r}\right)\right|^{2} \mathbf{1}_{\left\{\left|Y_{s}^{r}\right|+\left|Z_{s}^{r}\right| \leqslant \kappa\right\}} \mathrm{d} s\right]^{\frac{1}{2}}=: B_{1}^{p} \\
& +C \sum_{r=p}^{N_{p}} \lambda_{r}^{p} \mathbb{E}\left[\int_{0}^{t} \mathbf{1}_{\left\{\left|Y_{s}^{r}\right|+\left|Z_{s}^{r}\right|>\kappa\right\}} \mathrm{d} s\right]^{\frac{1}{2}}=: B_{2}^{p} .
\end{aligned}
$$

Combining Markov inequality with the uniform square integrability of $Y^{n}$ and $Z^{n}$, we easily obtain that

$$
B_{2}^{p} \leqslant \frac{C}{\kappa} .
$$

For the term $B_{1}^{p}$, we combine the uniform convergence (on compact set) of $H_{r}$ to $H$, recall Lemma 4.1(iii), with the dominated convergence theorem, since $H_{r}$ and $H$ are bounded, to get that for all $\epsilon>0$ there exists $N_{\kappa, \epsilon} \in \mathbb{N}$ such that

$$
B_{1}^{p} \leqslant \epsilon \text { for all } p \geqslant N_{\kappa, \epsilon} \text {. }
$$

Combining 4.12 and 4.13, we then get

$$
\lim _{p} \mathbb{E}\left[\left|A_{1}^{p}\right|\right]=0 .
$$

Next, we compute, using Cauchy-Schwartz inequality and the uniform bound on $\left\|\Phi^{n}\right\|_{\mathscr{H}^{2}}$,

$$
\mathbb{E}\left[\left|A_{2}^{p}\right|\right] \leqslant C \sum_{r=p}^{N_{p}} \lambda_{r}^{p} \mathbb{E}\left[\int_{0}^{t}\left|H\left(s, X_{s}^{0, a}, Y_{s}^{r}, Z_{s}^{r}\right)-H\left(s, X_{s}^{0, a}, Y_{s}, Z_{s}\right)\right|^{2} \mathrm{~d} s\right]^{\frac{1}{2}}
$$

and we deduce

$$
\lim _{p} \mathbb{E}\left[\left|A_{2}^{p}\right|\right]=0
$$

from the continuity of $H$ and the strong convergence of $\left(Y^{r}, Z^{r}\right)$ to $(Y, Z)$. Finally we use the boundedness of $H$ and the strong convergence of ${ }^{p} \Phi$ to $\Phi$ to get

$$
\lim _{p} \mathbb{E}\left[\left|A_{3}^{p}\right|\right]=0 \text {. }
$$

Combining (4.14), 4.15) and (4.16) with 4.10 yields $\lim _{p} \mathbb{E}\left[\left|\mathcal{E}_{t}^{p}\right|\right]=0$. Eventually, we get that, for all $t \leqslant T$,

$$
Y_{t}=g\left(X_{T}^{0, a}\right)+\int_{t}^{T} f\left(s, X_{s}^{0, a}, Y_{s}, Z_{s}\right) \mathrm{d} s-\int_{t}^{T} Z_{s} \mathrm{~d} W_{s}-\int_{t}^{T} H\left(s, X_{s}^{0, a}, Y_{s}, Z_{s}\right) \Phi_{s} \mathrm{~d} s,
$$

which concludes the proof of Theorem 4.1. Let us remark that the previous equation allows us to consider a continuous version of the process $Y$. 
We conclude this section by giving the proof of Corollary 4.1 which is an interesting application of Theorem 4.1 to the well studied case of BSDEs for switching problems. Following our approach, the main question reduces now to find an appropriate continuous $H$ to describe the direction of reflection such that $H(\cdot) \Phi=\Psi$, compare 4.2 and (4.4).

\section{Proof of Corollary 4.1}

It is sufficient to define a continuous function $H$ on $\partial \mathcal{D}$, recall Remark 2.1. We have

$$
\mathcal{D}=\left\{y \in \mathbb{R}^{d}: y^{l}>\max _{j \in \mathcal{I}}\left(y^{j}-c^{l j}\right), l \in \mathcal{I}\right\},
$$

thus, $\overline{\mathcal{D}}$ is a non-compact convex polyhedron. We can remark that

$$
\overline{\mathcal{D}}^{0}:=\overline{\mathcal{D}} \cap\left\{y^{d}=0\right\}
$$

is, by abuse of notation, a compact convex polyhedron of $\mathbb{R}^{d-1}$ and so it is a convex polytope. Indeed, we have

$$
\overline{\mathcal{D}}^{0} \subset\left\{\left(y^{1}, \ldots, y^{d-1}\right) \mid y^{i} \in\left[-c^{i d}, c^{d i}\right], \forall i \in\{1, \ldots, d-1\}\right\} \neq \varnothing,
$$

since we have $c^{d i}+c^{i d}>0$ for all $1 \leqslant i \leqslant d-1$ due to the structure condition (4.5). We just have to define $H$ on $\partial \overline{\mathcal{D}}^{0}$ and then extend $H$ to $\partial \overline{\mathcal{D}}$ in this way: for all $(t, x, y, z) \in[0, T] \times \mathbb{R}^{k} \times \mathcal{D} \times \mathbb{R}^{d \times k}$, we define

$$
H(t, x, y, z):=H\left(t, x,\left(y^{1}-y^{d}, \ldots, y^{d-1}-y^{d}, 0\right), z\right) .
$$

Since $\overline{\mathcal{D}}^{0}$ is a convex polytope, then, by Krein-Milman theorem, it is the convex hull of its extremal points. We will define $H$ on all extremal points and then the value of $H$ on all facets

$$
\mathcal{C}^{l j}=\left\{y \in \partial \mathcal{D}^{0}: y^{l}=y^{j}-c^{l j}\right\}, \quad l, j \in \mathcal{I}, \quad l \neq j,
$$

will be defined by linear interpolations. Let us consider an extremal point $\left(\bar{y}^{1}, \ldots, \bar{y}^{d-1}\right)$ : we know that there exist $\left(l_{i}, j_{i}\right)_{i \in\{1, \ldots, d-1\}} \in\{1, \ldots, d\}^{2 \times(d-1)}$ such that

- $\left(l_{i}, j_{i}\right) \neq\left(l_{k}, j_{k}\right)$ when $i \neq k$,

- for all $i \in\{1, \ldots, d-1\}, \bar{y}^{l_{i}}=\bar{y}^{j_{i}}-c^{l_{i} j_{i}}$ where $\bar{y}^{d}=0$.

Then, we define $H\left(t, x,\left(\bar{y}^{1}, \ldots, \bar{y}^{d-1}, 0\right), z\right)$ as the orthogonal projection onto $\operatorname{span}\left(\left\{e^{l_{1}}, \ldots, e^{l_{d-1}}\right\}\right)$. To conclude it is sufficient to check that $H\left(t, x,\left(y^{1}, \ldots, y^{d-1}, 0\right), z\right)$ sends the vector $e^{l}-e^{j}$ to the vector $e^{l}$ when $\left(y^{1}, \ldots, y^{d-1}\right) \in \mathcal{C}^{l j}$ and to show the result only for extremal points. In order to do so, let us consider $\left(\bar{y}^{1}, \ldots, \bar{y}^{d-1}\right) \in \mathcal{C}^{l j}$ an extremal point: by the definition of $H$ we just have to show that $e^{j} \notin\left\{e^{l_{1}}, \ldots, e^{l_{d-1}}\right\}$ where we re-use previous notations. Let us prove it by contradiction: we assume that there exists $i \in\{1, \ldots, d-1\}$ such that

$$
j=l_{i} \text { and } \bar{y}^{l_{i}}=\bar{y}^{j_{i}}-c^{l_{i} j_{i}} .
$$

Moreover, we have $\left(\bar{y}^{1}, \ldots, \bar{y}^{d-1}\right) \in \mathcal{C}^{l j}$ so

$$
\bar{y}^{l}=\bar{y}^{j}-c^{l j} .
$$


By combining 4.18), 4.19) and the structure condition (4.5), we obtain

$$
\bar{y}^{l}=\bar{y}^{j}-c^{l j}=\bar{y}^{j_{i}}-\left(c^{l j}+c^{j j_{i}}\right)<\bar{y}^{j_{i}}-c^{l j_{i}},
$$

which is in contradiction with the definition of $\mathcal{D}$ given by 4.17).

\subsection{The case of discontinuous $H$}

In this section, we consider the case of a discontinuous direction of reflection on the boundary $\partial D$. We obtain an existence result for an obliquely reflected BSDE but the characterization of the reflecting part is somehow more involved, specially at the discontinuity point of $H$, where many directions of reflection are allowed at the limit. This too weak characterization leads a to non-uniqueness result as illustrated in the next paragraph. The limiting equation we are studying here is then

$$
\begin{gathered}
Y_{t}=g\left(X_{T}^{0, a}\right)+\int_{t}^{T} f\left(s, X_{s}^{0, a}, Y_{s}, Z_{s}\right) \mathrm{d} s-\int_{t}^{T} Z_{s} \mathrm{~d} W_{s}-\int_{t}^{T} \Psi_{s} \mathrm{~d} s, \quad t \in[0, T] \\
\Psi_{s} \in E\left(s, X_{s}^{0, a}, Y_{s}, Z_{s}\right) \text { and } Y_{s} \in \overline{\mathcal{D}} \quad \mathrm{d} \mathbb{P} \otimes \mathrm{d} s \text { a.e. }
\end{gathered}
$$

with $a \in \mathbb{R}^{q}$ and, for all $(t, x, y, z) \in[0, T] \times \mathbb{R}^{q} \times \overline{\mathcal{D}} \times \mathbb{R}^{d \times k}$,

$$
E(t, x, y, z):= \begin{cases}\bigcap_{\varepsilon>0} \operatorname{pos}(\{H(t, x, \tilde{y}, \tilde{z}) u \mid(\tilde{y}, \tilde{z} \in B((y, z), \varepsilon), u \in \partial \varphi(y)\}) & \text { if } y \in \partial \mathcal{D} \\ \{0\} & \text { if } y \in \mathcal{D},\end{cases}
$$

where $\operatorname{pos}\left(\left\{v_{i}\right\}\right)$ is the closure of the positive linear span of the family $\left\{v_{i}\right\}$, and $B(x, \varepsilon)$ is the closed Euclidean ball of center $x$ and radius $\varepsilon$.

Theorem 4.2. Assume that assumptions (AM)(i)-(v) hold. Then, there exists a solution in $\mathscr{S}^{2} \times \mathscr{H}^{2} \times \mathscr{H}^{2}$ to 4.20 .

Remark 4.3. When $H$ is continuous, we can easily show that

$$
E(t, x, y, z)=H(t, x, y, z) \partial \varphi(y)
$$

which is consistent with the result of Theorem 4.1.

Proof. The proof of Theorem 4.2 strongly follows the proof of Theorem 4.1. The arguments are similar from step 1 to step 5.a. We thus start directly the proof at step $5 . \mathrm{b}$ by studying the reflecting term. Since

$$
\mathbb{E}\left[\int_{0}^{T}\left|H\left(s, X_{s}^{0, a}, Y_{s}^{n}, Z_{s}^{n}\right) \nabla \varphi_{n}\left(Y_{s}^{n}\right)\right|^{2} \mathrm{~d} s\right] \leqslant C,
$$

we have, up to a subsequence, the following weak $L^{2}([0, T] \times \Omega)$-convergence:

$$
\Psi^{n}:=H\left(., X_{.}^{0, a}, Y_{.}^{n}, Z_{.}^{n}\right) \nabla \varphi_{n}\left(Y_{.}^{n}\right) \rightarrow \Psi, \quad \text { when } n \rightarrow+\infty .
$$


Using once again Mazur's Lemma, we know that there exists a convex combination of $\left(\Psi^{n}\right)_{n \in \mathbb{N}}$ converging strongly in $L^{2}([0, T] \times \Omega)$, namely

$$
{ }^{p} \Psi:=\sum_{r=p}^{N_{p}} \lambda_{r}^{p} \Psi^{r} \stackrel{p \rightarrow \infty}{\rightarrow} \Psi
$$

where $\lambda_{r}^{p} \geqslant 0$ for all $p \in \mathbb{N}$ and $p \leqslant r \leqslant N_{p}$, and $\sum_{r=p}^{N_{p}} \lambda_{r}^{p}=1$. As usual, the following combination

$$
\left({ }^{p} Y,{ }^{p} Z\right):=\sum_{r=p}^{N_{p}} \lambda_{r}^{p}\left(Y^{r}, Z^{r}\right)
$$

still converges to $(Y, Z)$ in $\mathscr{S}^{2} \times \mathscr{H}^{2}$ and, by strong convergence,

$\sum_{r=p}^{N_{p}} \lambda_{r}^{p} f_{r}\left(\cdot, X^{0, a}, Y^{r}, Z^{r}\right) \stackrel{L^{1}([0, T] \times \Omega, d t \otimes d P)}{\longrightarrow} f\left(\cdot, X^{0, a}, Y, Z\right)$ and $\int_{0}^{t}{ }^{p} Z_{s} \mathrm{~d} W_{s} \stackrel{\mathscr{L}^{2}}{\longrightarrow} \int_{0}^{t} Z_{s} \mathrm{~d} W_{s}$.

So we can pass to the limit into

$$
{ }^{p} Y_{t}=g\left(X_{T}^{0, a}\right)+\int_{t}^{T} \sum_{r=p}^{N_{p}} \lambda_{r}^{p} f_{r}\left(s, X^{0, a}, Y_{s}^{r}, Z_{s}^{r}\right) \mathrm{d} s-\int_{t}^{T}{ }^{p} Z_{s} \mathrm{~d} W_{s}-\int_{t}^{T}{ }^{p} \Psi_{s} \mathrm{~d} s
$$

to obtain that

$$
Y_{t}=g\left(X_{T}^{0, a}\right)+\int_{t}^{T} f\left(s, X_{s}^{0, a}, Y_{s}, Z_{s}\right) \mathrm{d} s-\int_{t}^{T} Z_{s} \mathrm{~d} W_{s}-\int_{t}^{T} \Psi_{s} d s, \quad \mathrm{~d} t \otimes \mathrm{d} \mathbb{P} \text { a.e. }
$$

To conclude we just have to study the direction of reflection. Since we have, for all $n \in \mathbb{N}$,

$$
\Psi_{t}^{n}:=H\left(t, X_{t}^{0, a}, Y_{t}^{n}, Z_{t}^{n}\right) \nabla \varphi_{n}\left(Y_{t}^{n}\right) \in H\left(t, X_{t}^{0, a}, Y_{t}^{n}, Z_{t}^{n}\right) \partial \varphi\left(\mathfrak{P}\left(Y_{t}^{n}\right)\right)
$$

and $\left(Y_{t}^{n}, Z_{t}^{n}\right) \stackrel{n \rightarrow \infty}{\rightarrow}(Y, Z) \mathrm{d} t \otimes \mathrm{d} \mathbb{P}$ a.e., then, for all $\varepsilon_{1}>0$ and $\varepsilon_{2}>0$, there exists $N$ (depending on $\omega$ ) such that, for all $n \geqslant N$,

$$
\Psi_{t}^{n} \in \operatorname{pos}\left(\left\{H\left(t, X_{t}^{0, a}, \tilde{y}, \tilde{z}\right) u \mid(\tilde{y}, \tilde{z}) \in B\left(\left(Y_{t}, Z_{t}\right), \varepsilon_{1}\right), \bar{y} \in B\left(Y_{t}, \varepsilon_{2}\right) \cap \overline{\mathcal{D}}, u \in \partial \varphi(\bar{y})\right\}\right),
$$

$\mathrm{d} t \otimes \mathrm{d} \mathbb{P}$ a.e. It implies that, for all $p \geqslant N$,

$$
{ }^{p} \Psi_{t} \in \operatorname{pos}\left(\left\{H\left(t, X_{t}^{0, a}, \tilde{y}, \tilde{z}\right) u \mid(\tilde{y}, \tilde{z}) \in B\left(\left(Y_{t}, Z_{t}\right), \varepsilon_{1}\right), \bar{y} \in B\left(Y_{t}, \varepsilon_{2}\right) \cap \overline{\mathcal{D}}, u \in \partial \varphi(\bar{y})\right\}\right)
$$

$\mathrm{d} t \otimes \mathrm{d} \mathbb{P}$ a.e. Finally we get that

$$
\Psi_{t} \in \bar{E}\left(t, X_{t}^{0, a}, Y_{t}, Z_{t}\right) \quad \mathrm{d} t \otimes \mathrm{d} \mathbb{P} \text { - a.e. }
$$


where

$\bar{E}(t, x, y, z):=\bigcap_{\varepsilon_{1}>0, \varepsilon_{2}>0} \operatorname{pos}\left(\left\{H(t, x, \tilde{y}, \tilde{z}) u \mid\left(\tilde{y}, \tilde{z} \in B\left((y, z), \varepsilon_{1}\right), \bar{y} \in B\left(y, \varepsilon_{2}\right) \cap \overline{\mathcal{D}}, u \in \partial \varphi(\bar{y})\right\}\right)\right.$.

When $y \in \mathcal{D}$ we can remark that $\partial \varphi(\bar{y})=0$ when $\bar{y} \in B\left(y, \varepsilon_{2}\right) \cap \overline{\mathcal{D}}$ with $\varepsilon_{2}$ small enough: thus we get $E(t, x, y, z)=0$. When $y \notin \mathcal{D}$, Let us show that

$$
\partial \varphi(y)=\bigcap_{\varepsilon_{2}>0}\left\{u \mid u \in \partial \varphi(\bar{y}), \bar{y} \in B\left(y, \varepsilon_{2}\right) \cap \overline{\mathcal{D}}\right\} .
$$

One inclusion is obvious, we will prove the other one. Let us consider $u \in \partial \varphi\left(y_{n}\right)$ for all $n \in \mathbb{N}^{*}$ with $y_{n} \in B(y, 1 / n) \cap \overline{\mathcal{D}}$ and let us show that $u \in \partial \varphi(y)$. For all $z \in \overline{\mathcal{D}}$ and $n \in \mathbb{N}$ we have

$$
u \cdot(z-y)=u \cdot\left(z-y_{n}\right)+u \cdot\left(y_{n}-y\right)
$$

and so

$$
\sup _{z \in \overline{\mathcal{D}}}(u \cdot(z-y)) \leqslant \sup _{z \in \overline{\mathcal{D}}}\left(u \cdot\left(z-y_{n}\right)\right)+u \cdot\left(y_{n}-y\right) \leqslant|u|\left|y_{n}-y\right|
$$

by definition of $\partial \varphi\left(y_{n}\right)$. Then, by taking $n \rightarrow+\infty$ in the previous inequality we get

$$
\sup _{z \in \overline{\mathcal{D}}}(u \cdot(z-y)) \leqslant 0
$$

which proves 4.21). This result implies that for any $(\tilde{y}, \tilde{z}) \in \mathbb{R}^{d} \times \mathbb{R}^{d \times k}$,

$$
\bigcap_{\varepsilon_{2}>0}\left\{H(t, x, \tilde{y}, \tilde{z}) u \mid \bar{y} \in B\left(y, \varepsilon_{2}\right) \cap \overline{\mathcal{D}}, u \in \partial \varphi(\bar{y})\right\}=\{H(t, x, \tilde{y}, \tilde{z}) u \mid u \in \partial \varphi(y)\},
$$

and so we finally get that $\bar{E}=E$ which concludes the proof.

A counter-example to uniqueness Inspired by Remark 4.4 in [15], we suggest the following counter-example to uniqueness in a non-smooth setting. The domain $\mathcal{D}$ is given by

$$
\mathcal{D}=\left\{y \in \mathbb{R}^{3} \mid y_{1} \geqslant 0 \text { and } y_{2}+y_{1} \geqslant 0\right\}
$$

Observe that $\partial \mathcal{D}=F_{1} \cup F_{2}$, where $F_{1}$ and $F_{2}$ are given by

$$
F_{1}=\left\{y \in \mathbb{R}^{3} \mid y_{1}=0 \text { and } y_{2} \geqslant 0\right\}, F_{2}=\left\{y \in \mathbb{R}^{3} \mid y_{1} \geqslant 0 \text { and } y_{1}+y_{2}=0\right\}
$$

and we denote by $G=F_{1} \cap F_{2}$, the corner of the domain. On $F_{1}$ we assume that the reflection is normal so that $H=I_{3}$, including points on $G$ where the outward cone of reflection if given by

$$
\mathcal{K}=\left\{y \in \mathbb{R}^{3} \mid y_{1} \leqslant 0, y_{2} \leqslant 0 \text { and } y_{2} \geqslant y_{1}\right\} .
$$


The direction of reflection is along the $y_{1}$ axis on $F_{2} \backslash G$ and is thus oblique, $H$ is constant but not equal to $I_{d}$. $H$ is thus discontinuous at the corner.

We consider a BSDE with the following data: $X=W, \xi=\left(0,0, X_{T}\right)^{\top}, f(t, x, y, z)=$ $-\left(z^{3}, z^{3}, 0\right)^{\top}$ is constant. Note that it satisfies the assumption (AM)(i)-(v). We give now two distinct solutions:

1. The first solution is given by $Y_{t}=\left(0,0, W_{t}\right)^{\top}, Z_{t}=(0,0,1)^{\top}$ and $\Psi_{t}=(-t,-t, 0)^{\top}$.

2. The second solution is given by $Y_{t}^{\prime}=\left(T-t,-(T-t), W_{t}\right)^{\top}, Z_{t}^{\prime}=(0,0,1)^{\top}$ and $\Psi_{t}^{\prime}=(-2 t, 0,0)^{\top}$.

\section{References}

[1] J.-F. Chassagneux, R. Elie, and I. Kharroubi. A note on existence and uniqueness for solutions of multidimensional reflected BSDEs. Electron. Commun. Probab., $16: 120-128,2011$.

[2] J.-F. Chassagneux and A. Richou. Obliquely reflected BSDEs. arXiv:1710.08989v1.

[3] J. Cvitanic and I. Karatzas. Backward stochastic differential equations with reflection and Dynkin games. The Annals of Probability, pages 2024-2056, 1996.

[4] T. De Angelis, G. Ferrari, and S. Hamadène. A note on a new existence result for reflected BSDEs with interconnected obstacles. arXiv:1710.02389v1.

[5] C. Dellacherie and P.-A. Meyer. Probabilités et potentiel. Chapitres V à VIII, volume 1385 of Actualités Scientifiques et Industrielles /Current Scientific and Industrial Topics]. Hermann, Paris, revised edition, 1980. Théorie des martingales. [Martingale theory].

[6] N. El Karoui, C. Kapoudjian, É. Pardoux, S. Peng, and M.-C. Quenez. Reflected solutions of backward SDE's, and related obstacle problems for PDE's. the Annals of Probability, pages 702-737, 1997.

[7] A. M. Gassous, A. Răşcanu, and E. Rotenstein. Multivalued backward stochastic differential equations with oblique subgradients. Stochastic Processes and their Applications, 125(8):3170-3195, 2015.

[8] A. Gégout-Petit and É. Pardoux. Équations différentielles stochastiques rétrogrades réfléchies dans un convexe. Stochastics Stochastics Rep., 57(1-2):111-128, 1996.

[9] D. Gildbarg and N.S. Trudinger. Elliptic Partial Differential Equations, volume 1. 1977.

[10] S. Hamadène, J.-P. Lepeltier, and S. Peng. BSDEs with continuous coefficients and stochastic differential games. In Backward stochastic differential equations, pages 115-128. Harlow: Longman, 1997. 
[11] S. Hamadène and J. Zhang. Switching problem and related system of reflected backward SDEs. Stochastic Processes and their applications, 120(4):403-426, 2010.

[12] S. Hamadène and J. Zhang. Switching problem and related system of reflected backward SDEs. Stochastic Process. Appl., 120(4):403-426, 2010.

[13] Y. Hu and S. Tang. Multi-dimensional BSDE with oblique reflection and optimal switching. Probab. Theory Related Fields, 147(1-2):89-121, 2010.

[14] N. Kazamaki. Continuous exponential martingales and BMO, volume 1579 of Lecture Notes in Mathematics. Springer-Verlag, Berlin, 1994.

[15] P.-L. Lions and A.-S. Sznitman. Stochastic differential equations with reflecting boundary conditions. Comm. Pure Appl. Math., 37(4):511-537, 1984.

[16] J. Ma and J. Zhang. Path regularity for solutions of backward stochastic differential equations. Probab. Theory Relat. Fields, 122(2):163-190, 2002.

[17] R. Martyr. Finite-horizon optimal multiple switching with signed switching costs. Math. Oper. Res., 41(4):1432-1447, 2016.

[18] D. Nualart. The Malliavin calculus and related topics. Probability and its Applications (New York). Springer-Verlag, Berlin, second edition, 2006.

[19] É. Pardoux and S. G. Peng. Adapted solution of a backward stochastic differential equation. Systems Control Lett., 14(1):55-61, 1990.

[20] É. Pardoux and A. Răşcanu. Stochastic differential equations, Backward SDEs, Partial differential equations, volume 69. Springer, 2014.

[21] S. Peng. Monotonic limit theorem of BSDE and nonlinear decomposition theorem of Doob-Meyers type. Probability theory and related fields, 113(4):473-499, 1999.

[22] S. Ramasubramanian. Reflected backward stochastic differential equations in an orthant. Proc. Indian Acad. Sci. Math. Sci, 112(2):347-360, 2002.

[23] W. Schachermayer. A characterisation of the closure of $H^{\infty}$ in BMO. In Séminaire de Probabilités, XXX, volume 1626 of Lecture Notes in Math., pages 344-356. Springer, Berlin, 1996. 